\title{
Radio-loud ROSAT sources near the North Ecliptic Pole
}

\author{
W. Brinkmann ${ }^{1}$, M. Chester ${ }^{2}$, R. Kollgaard ${ }^{3}$, E. Feigelson ${ }^{4}$, W. Voges ${ }^{1}$, and P. Hertz ${ }^{5}$ \\ 1 Max-Planck Institut für extraterrestrische Physik, P.O. Box 1603, D-85740 Garching, Germany \\ 2 Dept. of Physics, Bucknell University, Lewisburg, PA 17837, U.S.A. \\ 3 Fermi National Accelerator Laboratory, Batavia, IL 60510, U.S.A. \\ 4 Dept. of Astronomy and Astrophysics, Pennsylvania State University, University Park, PA 16802, U.S.A. \\ ${ }^{5}$ E.O. Hulburt Center for Space Research, Naval Research Laboratory, Washington DC 20375-5320, U.S.A.
}

Received March 6; accepted August 6, 1998

\begin{abstract}
A deep and large-area survey of the North Ecliptic Pole region was made with the ROSAT All-Sky Survey and the VLA to elucidate the population of radioloud extragalactic objects. A region of 29.3 square degrees was surveyed with sensitivities around $510^{-14} \mathrm{erg} \mathrm{s}^{-1}$ $\mathrm{cm}^{-2}$ in the soft X-ray band and $1 \mathrm{mJy}$ at $1.5 \mathrm{GHz}$. Optical counterparts were sought on digitized Schmidt plates from POSS-I and II. Seventy-four reliable RASSVLA sources were found.

The sample is a heterogeneous mixture of Seyfert galaxies, quasars, BL Lac objects, galaxy clusters and groups containing a radio galaxy. Optical magnitudes range from $B \simeq 14$ to $B>22$. Three results are noteworthy: (1) the bimodality in the ratio of radio to optical emission seen in optically- and X-ray-selected AGN samples is also evident in the ROSAT-VLA objects; (2) X-ray and radio selection is an effective method for locating poor galaxy clusters and groups; and (3) a considerable population of optically faint but X-ray/radio-bright objects is present. This last group may be either distant clusters with radio galaxies or "red quasars".
\end{abstract}

Key words: galaxies: active — clusters: general - quasars: general - X-rays: general — radio continuum: galaxies

\section{Introduction}

The ROSAT All-Sky Survey (RASS) provides unique opportunities for large-area multi-frequency studies of extragalactic objects. The catalog of $\sim 80000$ sources which resulted from the recent RASS-II processing comprises the deepest soft X-ray $(0.1-2.4 \mathrm{keV})$ sample yet obtained (Voges et al. 1996), reaching a sensitivity of a

Send offprint requests to: W. Brinkmann,

e-mail: wpb@rzg.mpg.de few $\times 10^{-13}$ erg $\mathrm{cm}^{-2} \mathrm{~s}^{-1}$. Previous studies based on X-ray surveys such as the HEAO-1 Large Area Sky Survey (Wood et al. 1984) and the Einstein Extended Medium Sensitivity Survey (Gioia et al. 1990; Stocke et al. 1991) showed that the majority of X-ray-selected objects are extragalactic. In particular, active galactic nuclei (AGN) comprised 55 per cent of the EMSS, including 4 per cent BL Lacertae objects; normal or cooling flow galaxies another 3 per cent, and clusters of galaxies 13 per cent (Maccacaro et al. 1994).

While the task of optical identification of the entire RASS catalog will take years, special attention has been paid to the regions of the ecliptic poles which have unusually long exposures. As the ROSAT satellite carried out scans on great circles along ecliptic latitudes, the two ecliptic poles were observed for half a year once per orbit for up to 32 secs each time. Therefore the total exposure of the sky region around the North Ecliptic Pole (NEP), located at $\alpha=18^{\mathrm{h}} 00^{\mathrm{m}}$ and $\delta=66^{\circ} 30^{\prime}$, reached values of $\lesssim 40000 \mathrm{~s}$ (falling off with ecliptic latitude as $\sim$ arc$\sin (1 / \cos (\lambda))$, compared to the typical exposure for the RASS of $\lesssim 400 \mathrm{~s}$ near the ecliptic equator. The deep ROSAT pointings with the Position Sensitive Proportional Counter (PSPC) on the NEP (Bower et al. 1996) reached a total exposure of $\sim 79 \mathrm{ksec}$, but only data from the innermost 15.5 arcmin radius were analyzed (to minimize spurious detections due to the rib structure of the PSPC window). Thus the Survey observations of the NEP region still represent one of the deepest and spatially most extensive X-ray exposures of the sky ever performed.

Deep ROSAT pointings indicate the X-ray population expected around the NEP. For example, in eight PSPC pointings of the second Lockman hole (limiting flux of $1.710^{-14} \mathrm{erg} \mathrm{cm}^{-2} \mathrm{~s}^{-1}$ ), Carballo et al. (1995) identified 51 of 89 sources of which $\sim 50$ per cent are AGN and another $\sim 30$ per cent are galaxies or clusters of galaxies. In the similarly deep ( $\gtrsim 11^{-14} \mathrm{erg} \mathrm{cm}^{-2} \mathrm{~s}^{-1}$ ) but smallarea pointings centered on the North Ecliptic Pole, Bower 
et al. (1996) detected 20 sources and found a higher fraction of AGN, including $\sim 60$ per cent QSOs, a Seyfert 2 and a BL Lac object, as well as a cluster and two unidentified sources. Georgantopoulos et al. (1996) reported on a series of five deep $\left(\sim 310^{-15} \mathrm{erg} \mathrm{cm}^{-2} \mathrm{~s}^{-1}\right)$ PSPC pointings of fields chosen from the ultraviolet excess (UVX) survey of Boyle et al. (1990). Of the identified sources (145 of 194), 74 per cent are AGN (including QSOs and Seyfert 1 galaxies), another 13 per cent are galaxies, plus one cluster. As some AGN lie in clusters of galaxies, some confusion between the classes is probably present (Pierre et al. 1994).

Another approach to analyzing the extragalactic content of the X-ray source population is the cross-correlation of deep X-ray surveys with radio surveys. A primary advantage is the substantial improvement in precision of source positions, which in turn permits cross-correlations with faint optical surveys. Broad-band properties can then be determined prior to a comprehensive optical spectroscopic program, and the effort required for the latter is substantially reduced. Simultaneous X-ray- and radioselection produces exclusively extragalactic samples which can give insight into nonthermal processes and unified theories of AGN. Deep radio surveys (Windhorst et al. 1985; Thuan et al. 1992; Benn et al. 1993) and Hamilton \& Helfand's (1993) cross-correlation of deep Einstein IPC pointings and VLA observations suggest that a population of faint radio-emitting starburst galaxies could provide the necessary flux and spectral hardness to make up the faint end of the XRB. However, this suggestion is not confirmed by the ROSAT-ATCA study of the QSF3 field by Boyle et al. (1993).

In previous papers (Brinkmann et al. 1994; Brinkmann et al. 1995 (Paper I) and Brinkmann et al. 1997 (Paper II)), we reported cross-correlations of the RASS with large scale radio surveys. Brinkmann et al. (1994) compared the RASS source list with the Molonglo Reference Catalog of $408 \mathrm{MHz}$ radio sources (Large et al. 1991). Of the 546 coincidences found, 433 objects were previously spectroscopically identified. The radio - to - X-ray properties of this sample are discussed with an emphasis on implications for AGN unification schemes. Papers I and II compared the RASS sample with a source list generated from the $5 \mathrm{GHz}$ Green Bank survey of the northern sky (Condon et al. 1989). This radio survey covers most of the northern sky, from $0 \operatorname{deg} \leq \delta_{1950} \leq+75 \mathrm{deg}$ with a limiting sensitivity of $f_{\mathrm{r}} \gtrsim 20 \mathrm{mJy}$. The fraction of previously identified sources is much lower in this cross-correlation; of the 2127 coincidences, only 617 were previously known and their properties are discussed in Paper I. All objects were studied in follow-up observations with the VLA to obtain core fluxes and arc sec positions (Laurent-Muehleisen et al. 1997) and the radio - to - X-ray properties of the large sample of unidentified extragalactic sources are discussed in Paper II.
The X-ray spectra, optical colors and morphologies, and spectral energy distributions of the ROSAT - Green Bank (RGB) sources indicate that most of the unidentified sources are quasars, although some BL Lacs, radio galaxies, Seyferts and clusters are undoubtably present. However, their properties differ subtly from those of brighter radio-loud AGN such as the 1 Jy catalog (Kühr et al. 1979). These differences reflect the fact that the majority of the new RGB sources have broad-band properties between those of traditional radio-selected and X-ray-selected AGN. This is clearly shown among the RGB BL Lac objects (Laurent-Muehleisen et al. 1998). Deeper X-ray and radio surveys also offer the possibility of revealing an AGN population with radio properties intermediate between the traditional radio-loud AGN (PKS and 1 Jy samples) and radio-quiet AGN.

The plan of the paper is as follows. Section 2 discusses the multifrequency observations. Sections 2.1 summarizes the radio observations, more fully presented in Kollgaard et al. (1994) and Hertz et al. (1994). Section 2.2 describes the cross-correlations with COSMOS- and APMgenerated catalogs to provide optical characteristics. In Sect. 2.3 the ROSAT observations and details of the data analysis (including source detection and X-ray flux computation) are presented. Section 3 discusses the selection of sources for the multifrequency catalog and presents the catalog as three tables divided according to reliability of the X-ray-radio match and source morphology. Section 4 provides additional information on two subsets of sources. Section 4.1 presents X-ray spectral fits for the bright X-ray sources in the catalog. Section 4.2 describes the selection of candidate groups and clusters of galaxies, including individual source notes and radio-optical maps. Section 5 presents the broad-band properties of the sample, including the X-ray and radio flux distributions (Sect. 5.1), radio-to-optical and optical-to-X-ray spectral indices (Sect. 5.2), distribution of radio-to-optical spectral index (Sect. 5.3), and X-ray-to-radio and optical-toradio flux ratios (Sect. 5.3). Comparisons to the results of Papers I and II are made throughout Sect. 5. The conclusions are summarized in Sect. 6.

\section{Data sources and analysis procedures}

\subsection{The radio data}

In anticipation of the deep ROSAT exposure of the NEP, $1.5 \mathrm{GHz}$ continuum observations of a region of 29.3 square degrees centered on the NEP were carried out with the NRAO Very Large Array ${ }^{1}$ in 1990 and 1991. One hundred fourteen contiguous fields were observed in the $\mathrm{C}$ configuration giving an effective resolution around $12^{\prime \prime}$. The survey is sensitive to $1-2 \mathrm{mJy}$, with the value dependent on

\footnotetext{
1 The NRAO is operated by Associated Universities, Inc. under cooperative agreement with the National Science Foundation.
} 
location with respect to the field centers and field exposure times. A catalog of 2435 sources was generated, with total flux densities ranging from 0.3 to $1000 \mathrm{mJy}$ and positions for most objects accurate to at least $2^{\prime \prime}$. The catalog and the survey methodology are given by Kollgaard et al. (1994), and the individual fields are displayed by Hertz et al. (1994). This survey was several fold more sensitive, and produced an order of magnitude more sources, than previous radio surveys of the NEP region (e.g. Loiseau et al. 1988; Lacy et al. 1992; Lacy et al. 1995 and references therein), with sensitivities similar to the recent NVSS (Condon et al. 1998) and FIRST (Becker et al. 1995) VLA surveys.

\subsection{The optical data}

Optical candidates of RASS-VLA sources, their magnitudes and morphological classifications (starlike or galaxy) were selected primarily from a catalog of objects obtained from COSMOS scans of the second Palomar Observatory Sky Survey (POSS-II) plates provided by W.L.W. Sargent (CalTech). The catalog was created through a collaboration between the Naval Research Laboratory (NRL) and the Royal Observatory, Edinburgh (ROE) in support of the ROSAT mission (Yentis et al. 1992). Three blue (IIIa-J and GG395 filter) plates (PSIIJ071, PSIIJ102, and PSIIJ103) and two red (IIIa-F and RG610 filter) plates (PSIIF070 and PSIIF103) were available for candidate selection. Since the red plates covered only about twothirds of the region of the RASS-VLA survey, they were used mainly in cases of ambiguous classification of sources. Typical limiting magnitudes of the POSS-II plates are $B_{\mathrm{J}}=22.5 \mathrm{mag}$ and $R_{\mathrm{C}}=20.8 \mathrm{mag}$; the variation from plate to plate is $\pm 0.4 \mathrm{mag}$ (Reid et al. 1991). Standard star calibrations of individual plates yield photometric accuracies of a few tenths to $0.5 \mathrm{mag}$. However, resolved and/or bright objects pose certain problems and discrepancies with published magnitudes can exceed these values. The star-galaxy separation is expected to be reliable to a limit of $B \sim 21.0-21.5$ (MacGillivray \& Stobie 1984), although bright nearby galaxies pose special problems for the recognition of and integration over their full extent. Magnitudes for brighter objects were obtained from the NASA Extragalactic Database (NED) when available. Astrometric calibration of COSMOS-scanned plates is performed with the FK4 catalog; a conservative estimate of the positional accuracy is $1^{\prime \prime}$ (MacGillivray, private communication).

Additional candidates, magnitudes and classifications were selected from a catalog of objects obtained from POSS-I O and E plates digitized with the Automatic Plate Measuring (APM) machine at 1.0" resolution (McMahon 1991). The catalog was provided to us by R.G. McMahon. We initially consulted the APM catalog to fill in a small survey region covered only by a POSS-II red plate.

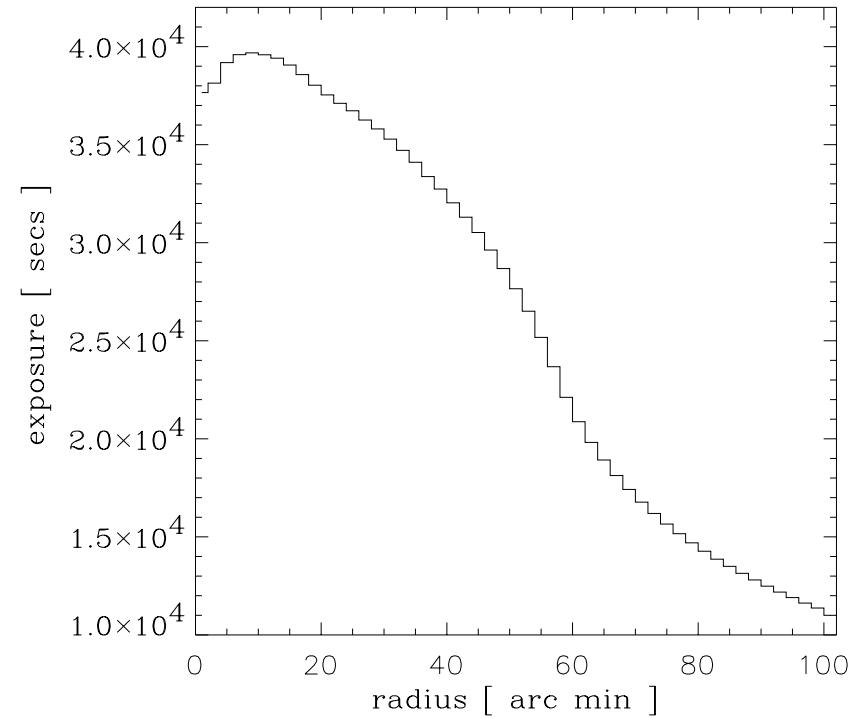

Fig. 1. Average Survey exposure in sec for the central field centered on the NEP as function of the radial distance from the geometrical center of the Survey exposure map

Although the APM catalog is not as deep as the COSMOS catalog, we found the APM catalog to be more consistent with published magnitudes and have used this to calibrate the magnitudes from the COSMOS plates (Sect. 3.2). The photometric accuracy of APM magnitudes is approximately \pm 0.5 mag (Laurent-Muehleisen et al. 1996; Gregg et al. 1996; see also McMahon \& Irwin 1992).

\subsection{The X-ray data}

The ROSAT All-Sky Survey (RASS) was conducted with the Position Sensitive Proportional Counter PSPC-C on board ROSAT continuously between August 1990 and February 1991. The mission and the instrument are described by Trümper (1983), Pfeffermann et al. (1986), and Aschenbach (1988). A preliminary source list (RASSI processing) has about 60000 sources (Voges 1992). In recent correlations of this list with radio catalogs (Brinkmann et al. 1994, Papers I, II) X-ray data were used which were processed in single $2^{\circ}$ survey strips. In this paper we utilize data summed up over the whole mission in the NEP region. The accumulated data were organized in nine $3^{\circ} \times 3^{\circ}$ fields centered on the NEP.

The exposure ranged from $\sim 40000 \mathrm{~s}$ near the NEP to $\sim 5000 \mathrm{~s} \sim 4.5^{\circ}$ off the NEP. In Fig. 1 we show the radially averaged exposure map of the central region around the NEP. The exposure times are corrected for instrumental vignetting and deadtime effects centered $\sim 3.5^{\prime}$ north of the nominal position of the NEP. Although the exposure is not exactly rotationally symmetric due to missing Survey strips, the curve clearly shows the $\arcsin (1 / \cos \lambda)$ behaviour at larger distances from the NEP, modified by the shadowing of the PSPC's support grid and the 


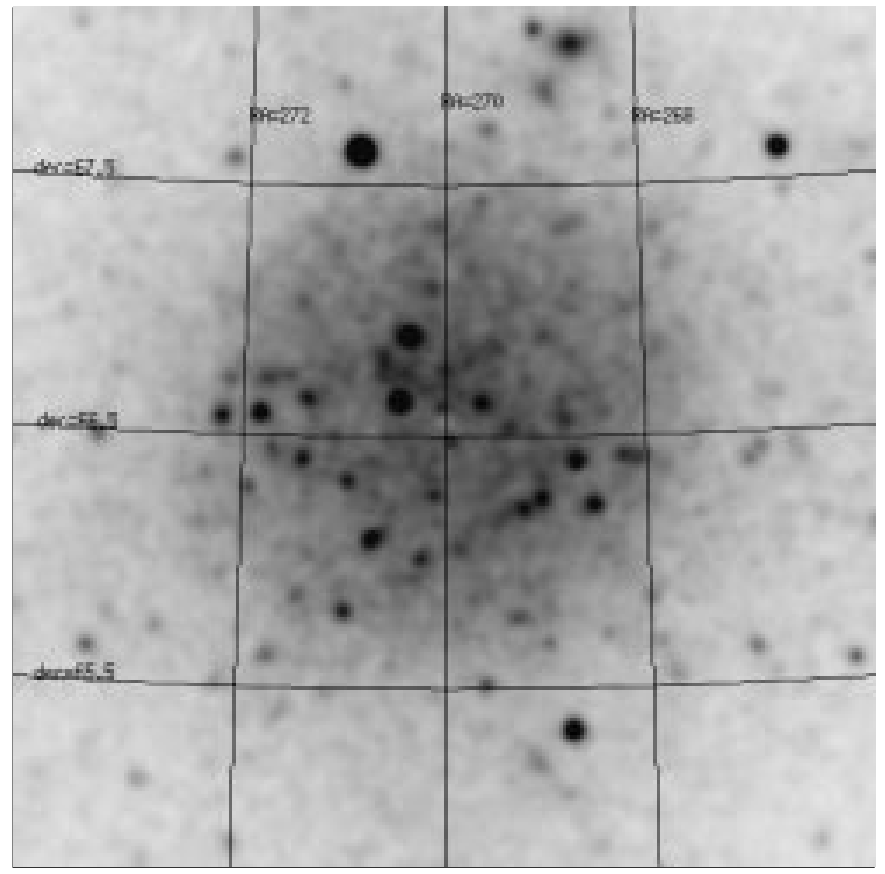

Fig. 2. ROSAT broad band photon image of the central $3^{\circ} \quad 3^{\circ}$ of the NEP field. Clearly visible is the slightly noncircular structure of the central parts and the "exposure hole" $3.5^{\prime}$ north of the NEP

non-uniform detector sensitivity. The local minimum at the center is caused by the continuous crossing of the central support grid and broadened by the slight offsets of the satellite orbit after scan reversals. This "exposure hole" can be seen in Fig. 2 where we plot a smoothed grey scale image of the central $\sim 3^{\circ} \times 3^{\circ}$ region around the NEP. The image demonstrates the high density of X-ray sources in the inner field and shows the slight asymmetry of the exposure.

\subsubsection{Source detection}

A catalog of X-ray positions and count rates was constructed from a source detection procedure built on standard commands available in the EXSAS environment and applied to each of the nine fields (Zimmermann et al. 1994). Two preliminary source detection procedures were used to generate locations of probable sources, which were used as input into a maximum likelihood procedure for the production of final source lists and properties.

1. A local detection algorithm was applied to the images with a binsize of $24^{\prime \prime}$ using broad band $(0.1-2.4 \mathrm{keV})$ and hard band $(0.5-2.0 \mathrm{keV})$ photons. This method counts photons in a sliding window adjusted to the size of the point-spread function, with background measured in the neighbouring pixels. The significance of excesses was measured with a likelihood ratio assuming Poisson statistics. A source was accepted for a likelihood ratio greater than 5 , which roughly corresponds

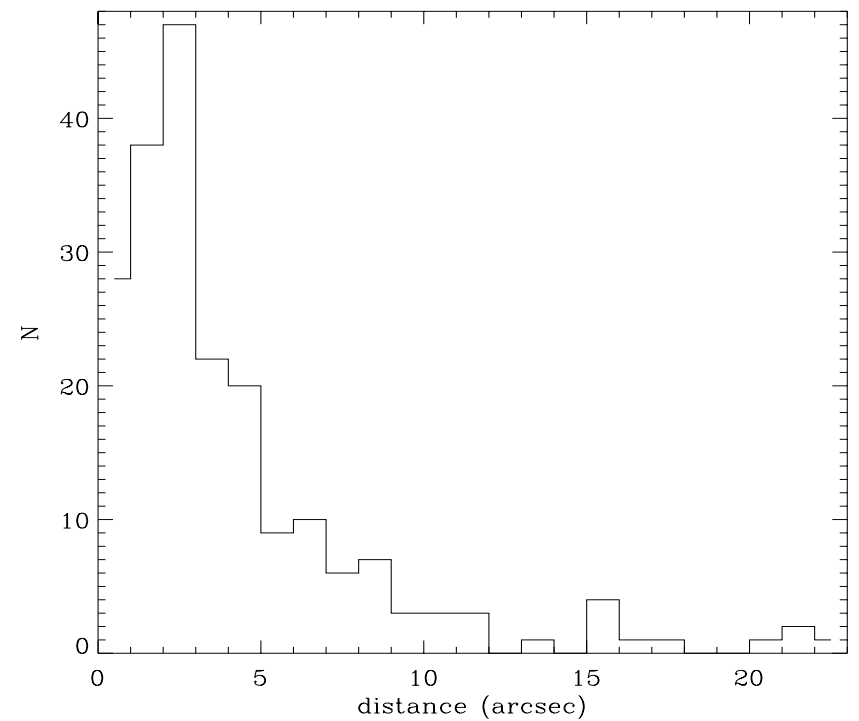

Fig. 3. Distance in arcsec between the same sources found from the total detector and the inner part only for the central $3^{\circ} 3^{\circ}$ field

to a Gaussian sigma of 2.3. See Cruddace et al. (1988) for a fuller description.

2. A global detection algorithm was developed as follows. Broad and hard band background images were created by cutting out sources found in step 1 with a likelihood ratio greater than $10(\sim 4.5 \sigma)$ from the images and applying a bi-cubic spline fit to the residuals after dividing by the exposure map to correct for vignetting. The resulting fit, re-multiplied by the exposure image, provides background values for a sliding window source detection algorithm as described above. A source was accepted for a likelihood ratio greater than 5 .

Four source lists were made by applying these two methods to the photon event data in the hard and total bands. The source lists were combined by merging sources lying within $3 \sigma$ positional errors and for overlaps in a circle with a diameter equal to the FWHM of the point-spread function $\left(60^{\prime \prime}\right.$ for the Survey), taking into account the possible extent (see Zimmermann et al. 1994, Sect. 5.2 for details). Source fluxes were measured in the two bands within a $5^{\prime}$ radius. Comparison of fluxes for sources in overlapping fields indicate that systematic errors of order $10 \%$ may be present in stronger sources, and larger errors for weak sources. Source lists and positions may also be incorrect in unusually crowded regions or for complex extended structures.

For most of the RASS it has been shown that the highest signal-to-noise is achieved with the full field of view of the detector for the source detection algorithm, even though the point spread function of the X-ray telescope degrades rapidly in the outer regions of the PSPC detector (Aschenbach, private communication). However, in regions of high source density like the NEP, the degraded point spread function causes an increase of the 


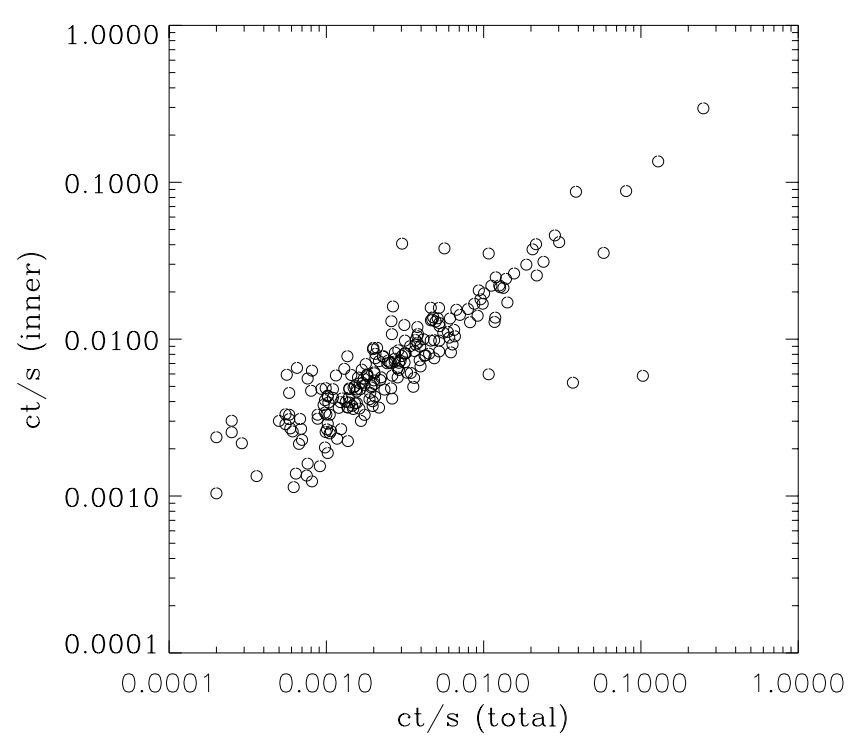

Fig. 4. Count rates of the same objects as found from the total detector and the inner part only

background flux through source confusion. We therefore repeated the source detection procedure for the deep central $3^{\circ} \times 3^{\circ} \mathrm{NEP}$ field using only photons from the inner part $\left(r_{\text {det }}<20^{\prime}\right)$ of the PSPC where the point spread function is uniform and narrow $\left(\lesssim 30^{\prime \prime}\right)$. Although this reduces the exposure by $2 / 3$ compared to the analysis using the full detector, we found that the number of sources increased from 266 sources to 583 sources when only the inner detector is used. The density of sources at the center of the field reaches $\simeq 100$ sources/square degree.

We have compared source properties obtained using the whole PSPC and the inner detector alone. Figure 3 shows that positional offsets are generally less than $10^{\prime \prime}$; exceptions are due to extended sources. We conclude that the internal accuracy of source positions is about $\sim 5^{\prime \prime}$ for unresolved sources. Even after accounting for systematic errors the accuracy should be better than the $\sim 18^{\prime \prime}$ (68\% level) found for the whole RASS (Voges et al. 1996) due to repeated exposure of the NEP region during the survey. For many sources, the inner detector analysis yielded more source photons, increased likelihood values and higher count rates (Fig. 4). The improvement is most pronounced for weaker sources. Outliers are again due to extended sources, indicating that source properties for these sources are highly dependent on the particular data analysis path chosen.

The result of our analysis, using the inner detector for the central $3^{\circ} \times 3^{\circ}$ field and the full detector in the remainder of the $9^{\circ} \times 9^{\circ}$ region around the $\mathrm{NEP}$, is the detection of more than 1700 sources. Due to the higher exposure, the central field contains 583 of these sources.

\subsubsection{Spectra and fluxes}

Only 18 sources have sufficient photons to permit direct estimation of the X-ray spectra. These spectra are discussed in Sect. 2.3.3. To estimate source fluxes for the remaining sources, we follow the analyses in Papers I and II and assume a power law spectrum with photon index $\Gamma=2.1$ and galactic absorption $N_{\mathrm{H}}$ (Dickey \& Lockman 1990) in the direction of the source. $N_{\mathrm{H}}$ varies relatively smoothly even on shorter angular scales (Elvis et al. 1994). The unabsorbed X-ray fluxes in the ROSAT band $f_{0.1-2.4 \mathrm{keV}}$ are computed using the source count rates and the energy-tocounts conversion factor for a power law fit (ROSAT AO2 technical appendix, 1991). The resulting fluxes will be inaccurate for sources with intrinsic absorption, different power law or complex spectra. We report here only errors due to the counting statistics.

Fluxes for sources in the inner field can be compared with those found by Bower et al. (1996) in their pointed observations. They report fluxes assuming a power law index of $\Gamma=2.0$ and a narrower $(0.5-2 \mathrm{keV})$ energy interval, giving a systematic reduction factor of $\sim 0.4$ compared to our values. Taking this into account, we find excellent agreement between the fluxes in most cases, indicating consistency between the data analyses for pointed observations and the approach followed here.

\subsubsection{X-ray bright objects}

Eighteen of the RASS sources in Tables $1-3$ have $>100$ photons, allowing simple spatial and spectral fitting of the PSPC data. Note that in some cases the objects are in crowded fields or in regions with strongly changing exposure so that the background subtraction may not be accurate. Table 4 presents the results of spectral fits using a power model with Galactic column densities. The columns give the source name, the number of vignettingand deadtime-corrected counts, the fitted power law photon index with its $1 \sigma$ error, the reduced $\chi^{2}$ for the fit, a likelihood parameter $\mathrm{ML}_{\text {ext }}$ that increases with the probability that the source is spatially extended, and remarks. In most cases the photon index is consistent with the expected $\Gamma=2.1$ (Sect. 2.3.2), albeit with large statistical errors.

\section{Identification of radio-loud RASS NEP sources}

\subsection{RASS-VLA correlation}

A list of radio-loud RASS sources was constructed by finding all unresolved RASS and VLA sources with offsets $\leq 25^{\prime \prime}\left(\leq 40^{\prime \prime}\right)$ in the central (outer) $3^{\circ} \times 3^{\circ}$ fields. For sources resolved in either band, a larger offset of $60^{\prime \prime}\left(100^{\prime \prime}\right)$ in the central (outer) $3^{\circ} \times 3^{\circ}$ fields was permitted to capture extended X-ray clusters containing a radio galaxy or 
Table 4. Power law fits to strong sources

\begin{tabular}{ccccccll}
\hline Name & Counts & $\begin{array}{c}N_{\mathrm{H} \text { gal }} \\
10^{20} \mathrm{~cm}^{-2}\end{array}$ & & $\Gamma$ & $\chi_{\text {red }}^{2}$ & ML $_{\text {ext }}$ & Comments \\
\hline $1732.9+6533$ & 114 & 3.3 & 1.9 & 0.4 & 1.2 & & QSO 4C65.21 \\
$1743.4+6342$ & 141 & 3.0 & 1.9 & 0.4 & 0.9 & 17 & (Fig. 5a) \\
$1748.5+7005$ & 306 & 4.0 & 2.7 & 0.2 & 0.9 & & QSO HB 1749+701 \\
$1748.6+6842$ & 125 & 4.2 & 1.4 & 0.6 & 0.8 & & Sy 1 Mkn 507 \\
$1751.4+6719$ & 104 & 4.5 & 2.9 & 0.3 & 0.8 & 15 & (Fig. 5d) \\
$1752.0+6551$ & 123 & 3.9 & 2.0 & 0.5 & 0.5 & & IC 1751+6551 \\
$1755.1+6519$ & 1169 & 3.7 & 2.6 & 0.1 & 0.7 & & IRAS F17549+6520 \\
$1756.5+6512$ & 153 & 3.7 & 1.3 & 0.9 & 0.9 & 26 & Gal CGCG 1756.3+6513 (Fig. 5f) \\
$1758.6+6637$ & 138 & 4.3 & 4.2 & 0.6 & 1.6 & & PN NG 6543; thermal \\
$1758.8+6349$ & 206 & 3.2 & 2.0 & 0.3 & 1.2 & & Sy 2 NGC 6552 \\
$1800.1+6636$ & 138 & 4.3 & 2.3 & 0.7 & 2.8 & & soft excess \\
$1800.5+6705$ & 100 & 4.3 & 3.2 & 0.4 & 1.2 & & \\
$1803.9+6548$ & 334 & 4.1 & 2.8 & 0.2 & 1.2 & 6.8 & \\
$1807.9+6617$ & 140 & 4.3 & 2.3 & 0.5 & 1.6 & & star \\
$1808.8+6634$ & 334 & 4.3 & 2.5 & 0.2 & 0.8 & & \\
$1815.4+6806$ & 104 & 5.1 & 1.9 & 0.5 & 0.7 & & \\
$1817.7+6824$ & 224 & 5.6 & 2.0 & 0.3 & 0.9 & & thermal? (Fig. 5j) \\
$1819.6+6857$ & 198 & 5.6 & 1.4 & 0.4 & 1.2 & 29 & \\
\hline
\end{tabular}

extended radio sources associated with an X-ray AGN. A total of 74 reliable matches were found. Table 1 gives the resulting RASS-VLA unresolved sources (28 matches) and Table 2 contains RASS-VLA sources where either the radio or X-ray source is extended or multiple (46 matches representing at least 37 separate radio sources).

The reliability of the associations was evaluated by repeatedly recorrelating the VLA catalog with X-ray source positions randomly shifted from the RASS positions by a range of angular distances, typically a few arc minutes. The simulations indicate that Tables 1 and 2 have high reliability with $\lesssim 10 \%$ spurious RASS-VLA associations. Table 3 contains possible additional sources (34 in number) with greater angular separations. Here the chance of spurious associations is around $50 \%$ in the central field and $20 \%$ in the outer fields. Most of the spurious listings will have faint $(1-2 \mathrm{mJy})$ radio flux densities.

The radio and X-ray data are summarized in the first 11 columns of the tables. Columns 1-5 are extracted from Kollgaard et al. (1994) and give the VLA source name, peak position in J2000, integrated flux density at $1.5 \mathrm{GHz}$ in mJy with its error. Secondary radio peaks which were individually cataloged in Kollgaard et al. are also listed. Column 6 gives the name of the corresponding ROSAT Survey source and in Col. 7 we list the positional offsets between the VLA and the X-ray sources in arcsec. Column 8 indicates whether the source lies in the inner $3^{\circ}$ (i) or outer region (o) of the RASS NEP field. Column 9 gives the likelihood value from the EXSAS processing. Columns 10 -11 present the $0.1-2.4 \mathrm{keV} \mathrm{X}$-ray flux (with error) in

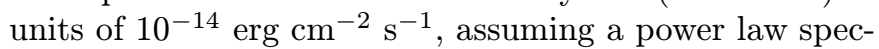
trum with photon index $\Gamma=2.1$ and Galactic absorption.

\subsection{Optical counterparts}

The RASS-VLA matches were correlated with optical catalogs produced from densitometric scans of blue plates from the Palomar Observatory Sky Surveys (POSS). As indicated in Sect. 2.2, two systems are used: APM scans of the POSS-I O plates and COSMOS scans of the POSS-II J plates. Optical positions within $4^{\prime \prime}$ of VLA radio positions were considered likely matches. The resulting objects are listed in Cols. $12-15$ of Tables $1-3$ with optical morphological class, optical minus radio positional offsets in arcsec, and estimated $B$ magnitudes. A few objects with offsets up to $8^{\prime \prime}$ are included when the identification is indicated in the literature or the radio source is extended. Additional nearby optical objects are given in the table notes with offset $(\Delta \alpha, \Delta \delta)$ in arcsec. As the radio sources in Table 2 are often extended, the absence of a listed optical counterpart is not a reliable indication that a counterpart is not present.

We found significant discrepancies, up to 3 magnitudes, between APM and COSMOS blue magnitudes and, where available, between both systems and published magnitudes. These differences are much greater than expected from the changes in emulsion-filter combinations used for POSS-I and II. We constructed a rough magnitude system by calibrating COSMOS measurements to APM magnitudes for stellar sources within $1^{\prime}$ of RASS-VLA sources. A linear regression between COSMOS MCOR values and $B_{\mathrm{APM}}$ gives $\mathrm{MCOR}=$ $(0.56 \pm 0.02) \times B_{\mathrm{APM}}+(-13.3 \pm 0.3)$. This formula was used to convert COSMOS measurements into magnitudes when an object was found on both plates. Values are rounded to the nearest 0.5 magnitude. However, many 
objects are found only in one of the APM or COSMOS catalogs, and a cross-calibration could not be performed in these cases. We caution that magnitudes reported in the tables are based on heterogeneous magnitude systems, and may suffer systematic uncertainties around $\Delta B \sim \pm 1$. When a source was not detected by either APM or COSMOS blue plates, a limiting magnitude of $B>22.5$ from the deeper COSMOS plates is adopted.

In Tables $1-3$, Col. 12 presents an optical morphology classification derived in most cases from the COSMOS object catalog: $\mathrm{E}=$ empty field; $\mathrm{F}=$ too faint to classify; $\mathrm{G}=$ galaxy; $\mathrm{Gp}=$ galaxy group; $\mathrm{S}=$ stellar. The Gp classification was made by us as described in Sect. 4.2. Columns 13-14 give the optical minus VLA positional offsets in arcsec. Column 15 gives approximate $B$ magnitudes derived as follows: $\mathrm{a}=\mathrm{APM}$ object catalog; $\mathrm{b}=$ COSMOS object catalog calibrated to APM magnitudes; $\mathrm{c}=$ COSMOS object catalog. The three objects with an "a" code occurred in the region where no blue POSS II plate was available (one case) or were not detected on the blue POSS II plate but were detected on the red plate and/or POSS I. Magnitudes without codes were obtained from the literature cited in the table notes. Table notes give crossidentifications and associated data obtained from the NASA Extragalactic Database (NED) or from VéronCetty \& Véron (1996), and indicate whether radio or $\mathrm{X}$-ray extended structure is present. Table notes also list nearby optical objects (from COSMOS or APM catalogs) with magnitudes and $(\Delta \alpha, \Delta \delta)$ in arcsec.

\section{Galaxy groups and clusters}

An important result of the RASS-VLA cross-correlation is the detection of a number of likely galaxy groups and clusters where the X-ray emission may derive from a hot intracluster medium. While most clusters reported from $\mathrm{X}$-ray surveys depend on X-ray extent and optical galaxy counts, we include radio extent as an alternative criterion, that is, the presence of extended or complex radio emission from a radio galaxy within the cluster. Studies with earlier X-ray satellites have established that a statistical link between X-ray and radio emission is present in clusters (Sarazin 1986 and references therein; but see e.g. Burns et al. 1994), perhaps the consequence of lobe confinement or feeding of the central AGN by the hot medium.

Ten sources in our study are definite or likely galaxy groups, six of which are known from previous optical or $\mathrm{X}$-ray surveys and four are proposed new identifications. Candidates were chosen by examining the morphology of RASS-VLA sources for which either the X-ray or radio emission is extended. We eliminated from consideration sources likely to be AGN, where an unresolved X-ray source coincides with the core of the radio morphology. Optical images were then examined for evidence of a group or cluster of galaxies. No formal galaxy count criterion was used. The ten group candidates exhibit a mix of morphological characteristics. In eight cases, the X-ray emission is extended in the RASS data. Conversely, all but one of the sources with extended X-ray emission satisfied our criteria for a candidate group. (The exception has only a marginally significant $\mathrm{ML}_{\text {ext }}$ value, see notes to Table 2.) At least three cases have compact radio emission rather than resolved lobes. In four cases, both the X-ray and radio emission are extended or have multiple components.

While our use of radio morphology finds new group candidates, we recovered some but not all of the groups found in previous X-ray studies of the NEP region. The Einstein Extended Medium Sensitivity Survey (EMSS) serendipitously discovered one cluster in our survey region (Henry et al. 1992). One of our group candidates, $1755.4+6803$, lies near the EMSS cluster's position, but the VLA source lies several arcminutes east of the main cluster emission and may not be related. Burg et al. (1992) analyzed a $50 \mathrm{ksec}$ PSPC pointing and all-sky scans, and identified a region of extended, low X-ray surface brightness (first reported by Hasinger et al. 1991) as a new cluster with a richness class of 0 and redshift $\sim 0.09$. This cluster, labeled NEPX1, along with six Abell clusters and other likely groups of similar redshift within $50 \mathrm{Mpc}$, suggest a supercluster is present. We find a RASS-VLA source (VLA 1801.5+6645) at NEPX1 which marginally fits our criteria for a group candidate (see notes to the figures, below); however, the X-ray source is not extended in the RASS data. Henry et al. (1995) used RASS observations of a large region around the NEP with an optical criterion to select galaxy groups. They identified four groups within the boundaries of our survey, two of which $(1756.5+6512$ and $1751.1+6531)$ we also select as groups. Both have unresolved radio sources and optical images suggesting the groups are $\mathrm{cD}$-dominated. Of the other two Henry et al. groups, one has a number of VLA sources within the apparent group boundaries, while the other may have one or two associated VLA sources. Bower et al. (1996) analyzed $80 \mathrm{ksec}$ of PSPC pointings and did not detect NEPX1, but noted that their detection algorithm would miss a cluster of such low X-ray surface brightness. They did detect a new, high-redshift $(z=0.57)$ cluster, RX J1801.7+6637, with an X-ray flux of $F_{\mathrm{x}}=1.2510^{-14} \mathrm{erg} \mathrm{cm}^{-2} \mathrm{~s}^{-1}$, near the limit of our survey and not detected by us in the X-ray or radio.

Of the four Abell clusters (Abell et al. 1989) which lie within the boundaries of our survey, two are associated with RASS-VLA sources: $1743.4+6342$ lies within the region of Abell 2280, and the triple VLA source $1819.6+6857,1819.6+6856$, and $1819.7+6856$ is a member of Abell 2304. X-ray emission for these sources has been previously reported from ROSAT data (Gioia et al. 1995; Gómez et al. 1997).

Due to the diversity of morphologies, we provide figures for each of the group candidates and discuss them 
individually. Figure 5 gives grey-scale optical images of each candidate group, produced with the Skyview utility provided by the HEASARC, overlaid with the radio contours of the VLA sources (Hertz et al. 1994). Contours levels are percentages of the radio peak chosen so that the least significant contour is approximately twice the noise and subsequent contours increase by factors of two; the highest contour is $99 \%$ of the radio peak. Except for $1801.5+6645$, which has a slightly larger field, the images are $4^{\prime} \times 4^{\prime}$ in size. The lowest contour level for each of the images is given in notes to the figures. Radio spectral indices are given in Kollgaard et al. (1994). Additional source information is given in the notes to Tables 2 and 3 .

\section{Notes to the figures: \\ 1743.4+6342 Fig. 5a:}

$8 \mathrm{C} 1743+637$. The radio position is in the outer regions of extended X-ray structure located predominantly to the south and associated with Abell 2280 (see Gioia et al. 1995). However, Lacy et al. (1993) suggest that the optical counterpart to the radio galaxy is at a much higher redshift. (See Table 4 for X-ray spectral fit.) The lowest radio contour level is at $2 \%$ of the peak value.

$\mathbf{1 7 4 9 . 8}+6824, \mathbf{1 7 4 9 . 8}+6823$ Fig. 5 b:

The centroid of the X-ray emission lies approximately midway between the VLA sources $1749.8+6824$ (MCG+11 $22-005)$ and $1749.8+6823$ (KUG1750+683A). The extended X-ray structure is complex with a pronounced "emission hole" on the single radio galaxy, 1749.9+6824 (CGCG1750.1+6825). Lowest radio contour level at $5 \%$ of the peak value.

1751.1+6531 Fig. 5c:

NGC 6505 (CGCG1751.1+6533). The radio source is centered on the maximum of the X-ray emission. Strong extended X-ray structures lie mainly in the north-western part of the image. The optical galaxy is an E/S0 with readily apparent halo, possibly a $\mathrm{cD}$ galaxy in the group or cluster. (See Henry et al. 1995). Lowest radio contour level at $30 \%$ of the peak value.

1751.4+6719, 1751.6+6719 Fig. 5d:

The X-ray centroid is between these two VLA sources, closest to $1751.4+6719$ which appears to be the core of a radio galaxy; $1751.6+6719$ and $1751.2+6719$ are the apparent lobes. The X-ray emission is circularly extended with 1751.2 just outside the main emission. (See Table 4 for X-ray spectral fit.) All three radio sources are shown in Fig. 5d with the lowest radio contour at $8 \%$ of the peak. $\mathbf{1 7 5 5 . 4 + 6 8 0 3}$ Fig. 5e:

We select a group candidate near the position of the EMSS cluster MS1754.9+6803. The RASS source spatially correlated with the VLA source is embedded in a relatively faint chain of emission. The main, very strong cluster emission lies several arcminutes west and has been identified with ZwC1754.5+6807 (Gioia \& Luppino 1994 and Henry, private communication). Thus our selection of this cluster may be coincidental. Lowest radio contour at $16 \%$ of peak value.

1755.7+6754 Fig. 5f:

The radio source is located in the outer part of relatively weak extended emission of $\sim 3^{\prime}$ size, elongated towards the southeast. The optical image shows a possible elliptical with readily apparent halo, perhaps a cD galaxy in a group or cluster. The lowest radio contour is at $12 \%$ of the peak value.

1756.5+6512 Fig. $5 \mathrm{~g}$ :

CGCG1756.3+6513. The radio source coincides with one of the peaks of very extended strong X-ray emission, mainly in a south-westerly direction. The optical galaxy is an E/S0 with readily apparent halo, possibly a cD galaxy in the group or cluster. (See Henry et al. 1995). (See Table 4 for X-ray spectral fit.) Lowest radio contour at $20 \%$ of peak value.

1801.5+6645 Fig. 5h:

Two VLA sources, 1801.5+6645 (unresolved, weak) and $1801.4+6643$ (possibly resolved, strong) lie near the unresolved RASS source. In the south the maximum of the X-ray emission coincides with the compressed contour lines to the south-east of $1801.4+6643$. The chain of optical objects north of $1801.5+6645$ follows a narrow structure of reduced X-ray emission. Burg et al. (1992) detected NEPX1, a region of low surface brightness X-ray emission in the vicinity of the VLA and RASS sources. They give optical identification of several associated galaxies. Note: the RASS-VLA source is listed in Table 3 because the $\mathrm{X}$-ray-radio offsets are greater than our criteria for unresolved sources (but within our criteria for extended or multiple-component sources). Lowest radio contour at $8 \%$ of the peak value.

\section{8+6536 Fig. 5i:}

The radio source coincides with a local maximum of a cross-like structure in X-rays. The strongest X-ray emission is found $\sim 1^{\prime}$ to the north-east of $1806.8+6536$, near the brighter, extended optical object. Lowest radio contour is at $8 \%$ of the peak value.

1819.6+6857, 1819.6+6856, 1819.7+6856 Fig. 5j:

$4 \mathrm{C} 68.21$ in Abell 2304. 1819.6+6857 is one of a sample of wide-angle tailed radio galaxies in Abell clusters analyzed by Gómez et al. (1997) as part of a study of substructure in cluster X-ray emission. (See also Owen et al. 1996 and references therein.) While the bulk of the cluster's Xray emission is a few arcminutes to the east, local $\mathrm{X}$-ray maxima trace closely the peaks of the radio contours. An 15.0 mag optical galaxy coincides with the apparent radio core, $1819.7+6856$. (See Table 4 for X-ray spectral fit.) Lowest radio contour at $0.5 \%$ of the peak value. 

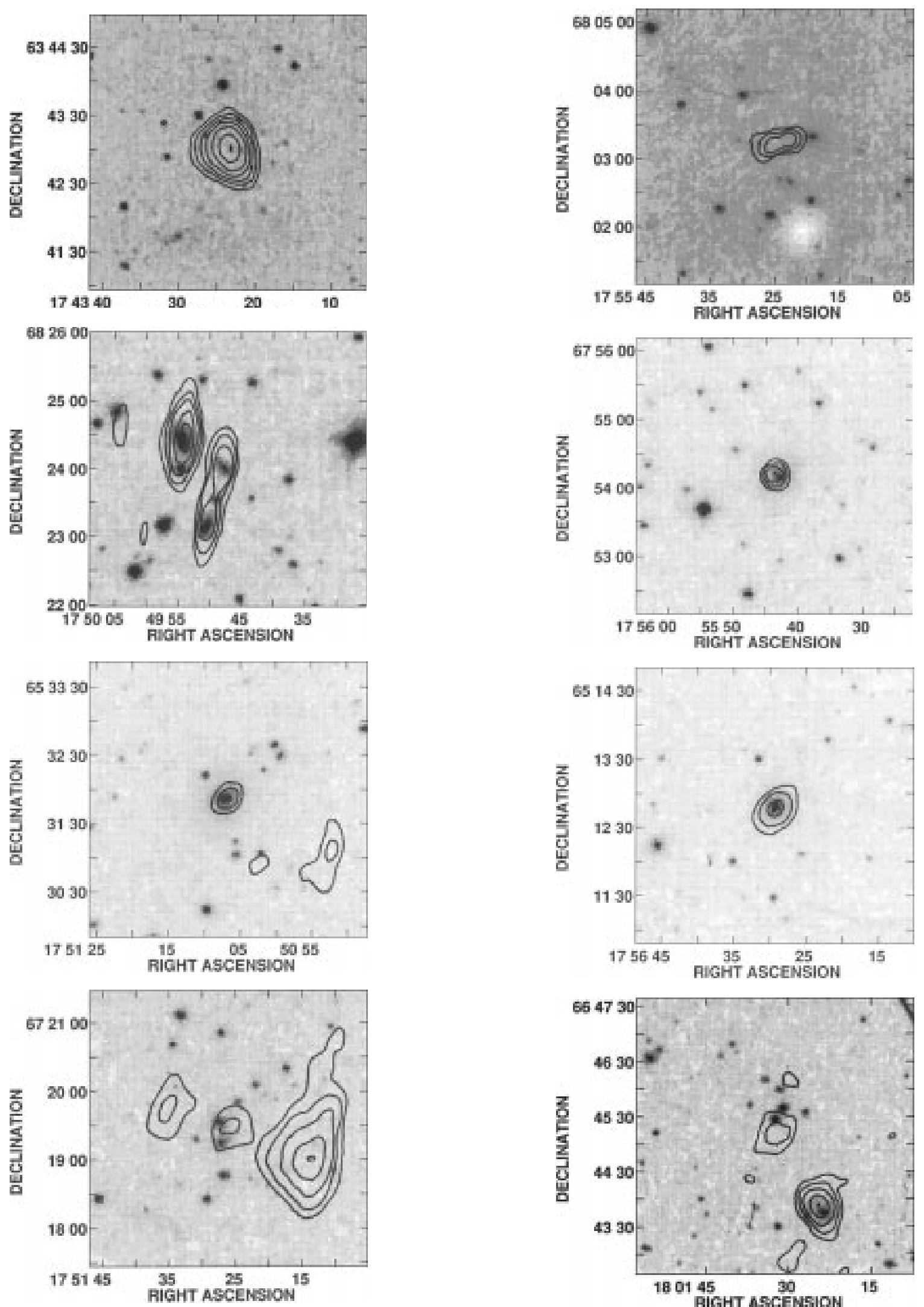

Fig. 5. Figures a-d), see text for notes

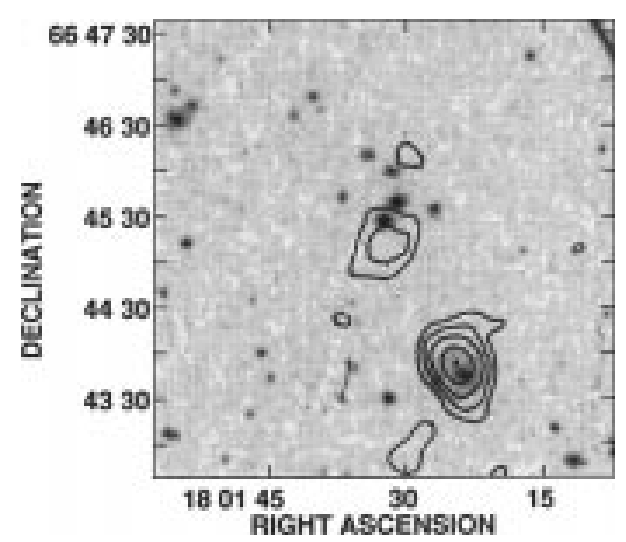

Fig. 5. Figures e-h), see text for notes 

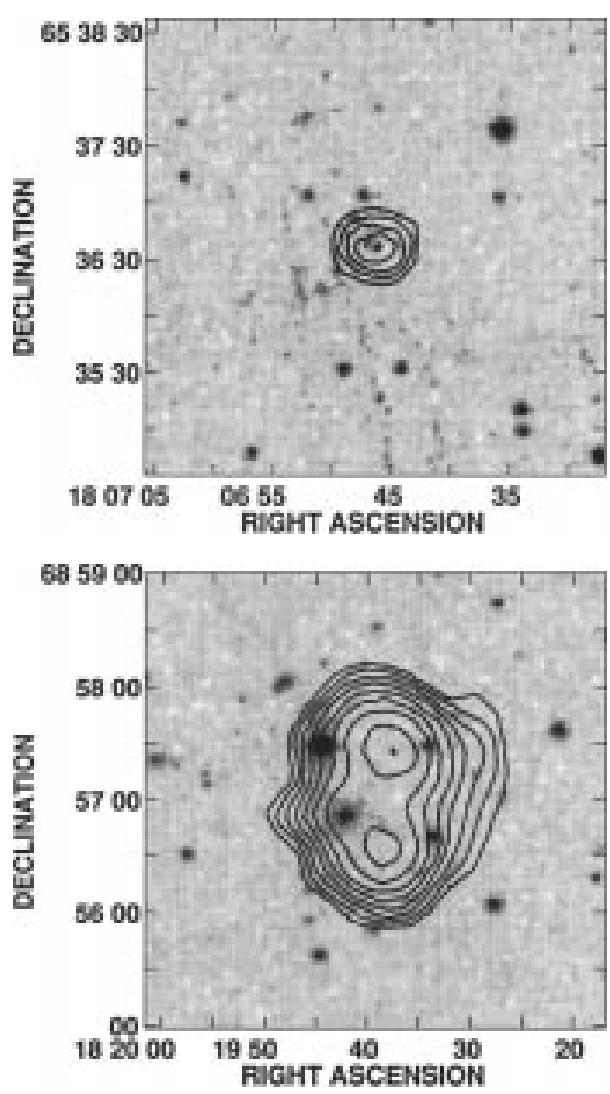

Fig. 5. Figures i-j), see text for notes

\section{Broad band source properties}

\subsection{Comparison with shallower radio-X-ray samples}

Papers I and II constructed a large sample of radio-loud X-ray objects from the correlation of the RASS survey with the $5 \mathrm{GHz}$ Green Bank survey. The resulting RGB sample is an order of magnitude less sensitive in both spectral bands than the NEP sample obtained here, although it covers a much larger area of the sky. The relationship between the two surveys is illustrated in Fig. 6; the RGB histograms are drawn from Fig. 4 of Paper II. Two features can be noted. First, while the X-ray fluxes of the NEP sources are confined to about 1.5 orders of magnitude ${ }^{2}$, the radio flux densities are spread over 3 orders of magnitude. Any correlations between the quantities must show considerable scatter. Second, no bimodality is seen in the radio flux density distribution as is seen, for example, in the optically-selected quasar samples of Strittmatter et al. (1980) and Kellerman et al. (1989). This result is consistent with the findings of Papers I and II which, however, did not significantly sample the faint end of the radioloud AGN population in either radio luminosity or the

\footnotetext{
${ }^{2}$ The distribution of X-ray fluxes here does not directly reflect the intrinsic $\log N-\log S$ curve for two reasons: the selection of radio-loud ROSAT sources may be X-ray fluxdependent, and the survey exposure is far from uniform (see Fig. 1).
}
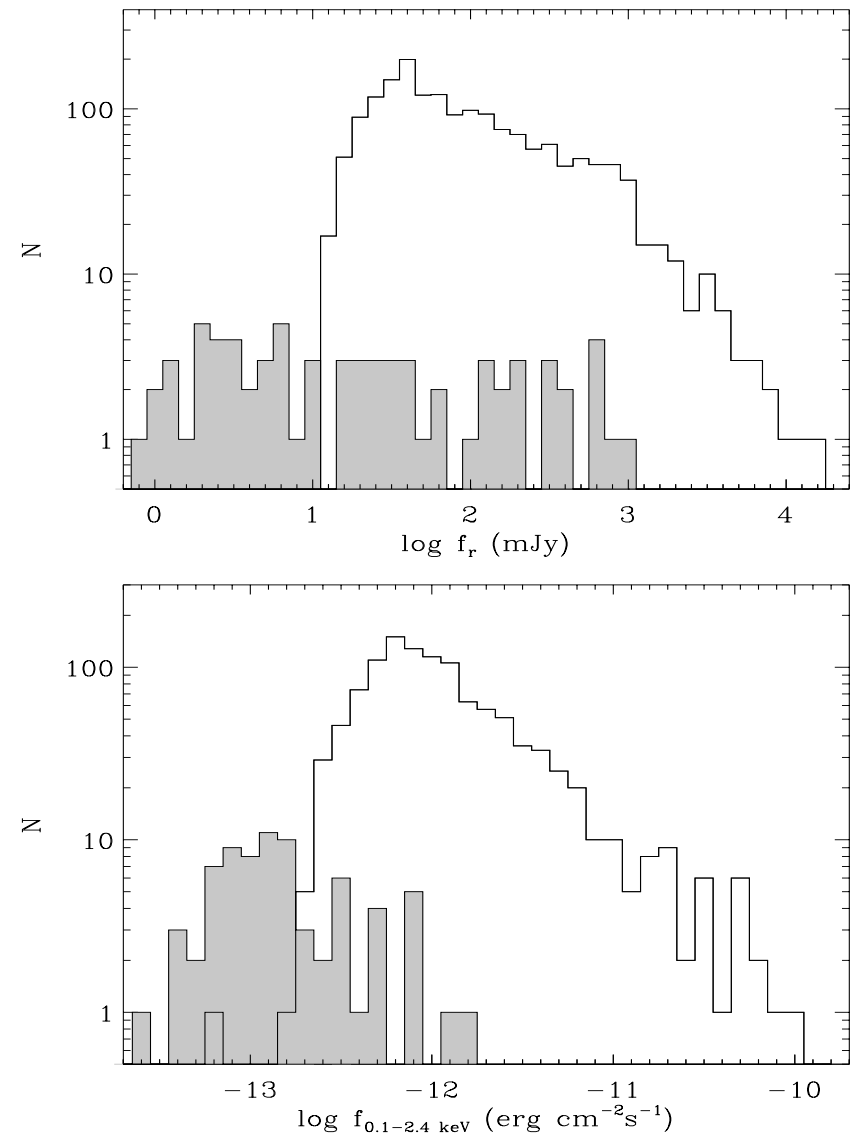

Fig. 6. Flux distribution of the NEP sources (shaded) compared to that of the ROSAT - 87 GB survey (open histogram)

ratio of radio-to-optical emission. Bimodality is evident in the NEP sample in the distribution of the ratio of radioto-optical emission, as measured by the radio-to-optical spectral index, $\alpha_{\text {ro }}$. As discussed in the next section, the observed bimodality is consistent with optically-selected quasar samples (e.g. Kellerman et al. 1989, 1994, and Stocke et al. 1992) and the X-ray-selected AGN sample of Della Ceca et al. (1994).

Figure 7 shows the integrated $0.1-2.4 \mathrm{keV}$ flux distribution of all RASS sources in the central $3^{\circ} \times 3^{\circ}$ field of the NEP and the distribution of the radio-detected subgroup. The radio-loud group has a slightly higher average X-ray flux than the parent population, but radio-loud sources are found at all X-ray flux levels. At the higher flux levels of the RGB samples, a similar uniform distribution of radio-loud objects is seen (Paper I, Fig. 6). The absolute detection rate in the NEP field is similar to that of other deep X-ray observations (de Ruiter et al. 1994; de Ruiter et al. 1997; Warwick \& Barber 1992) and considerably higher than for the RGB survey (Papers I and II). 


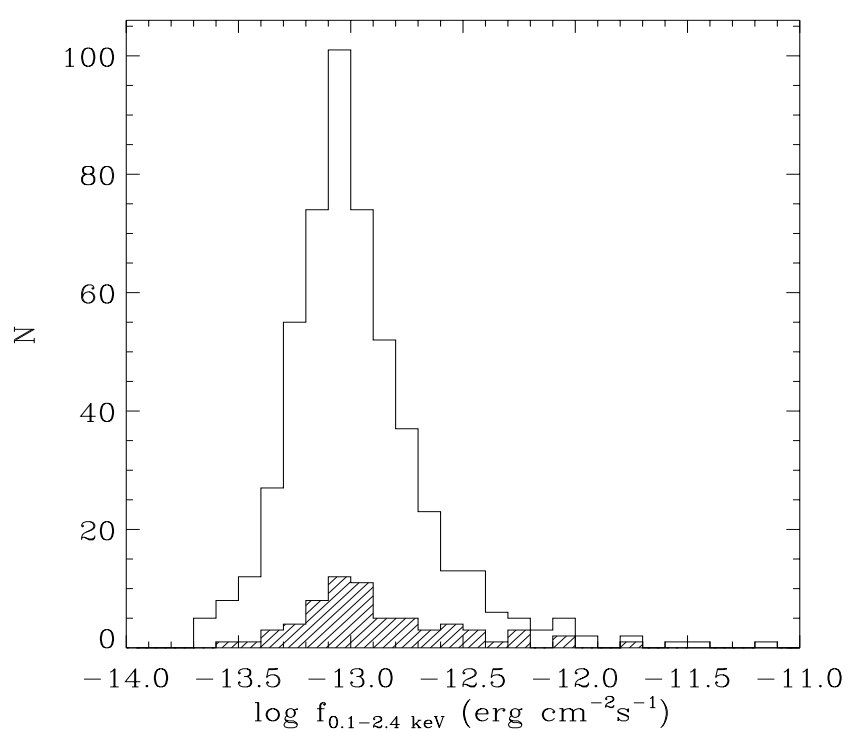

Fig. 7. X-ray flux distribution of all the NEP sources in the inner $3^{\circ} \quad 3^{\circ}$ field (open diagram) and the radio-loud subsample (hatched area)

\subsection{The $\alpha_{\text {ro }}-\alpha_{\text {ox }}$ diagram}

The broad-band $\alpha_{\text {ro }}-\alpha_{\text {ox }}$ color-color diagram has been extensively used to display broad-band spectral energy distributions and to classify extragalactic objects. The radio-to-optical index is defined as $\alpha_{\text {ro }} \equiv$ $\log \left(S_{\nu_{\text {rad }}} / S_{\nu_{\text {opt }}}\right) / \log \left(\nu_{\text {opt }} / \nu_{\text {rad }}\right)$ and the optical-to-X-ray index as $\alpha_{\text {ox }} \equiv-\log \left(S_{\nu_{\mathrm{x}}} / S_{\nu_{\mathrm{opt}}}\right) / \log \left(\nu_{\mathrm{x}} / \nu_{\text {opt }}\right)$. Here we use the radio fluxes at $\nu_{\mathrm{rad}}=1.5 \mathrm{GHz}$, the optical data at $4500 \AA$ and the X-ray data at $2 \mathrm{keV}$. While it has been widely asserted that different classes of objects occupy distinct locations in this diagram, we note that the boundaries can become less distinct as surveys become deeper in all bands (see Fig. 10 of Paper II). Nonetheless, the diagram is an effective tool for examining the spectral energy distributions of objects without redshifts such as the NEP population discussed here.

The $\alpha_{\text {ro }}-\alpha_{\text {ox }}$ diagram for the 74 sources in Tables 1 and 2 is shown in Fig. 8. It was constructed in a similar manner to Fig. 10 in Paper II with the following exception. In cases where the radio source appears to be multiple but the peaks were individually cataloged in Kollgaard et al. (1994), only the integrated flux density of the dominant component is used. The monochromatic X-ray fluxes at $2 \mathrm{keV}$ were computed from the observed count rate assuming a typical power law photon index $\Gamma_{\mathrm{x}}=2.1$ and absorption by the Galactic neutral hydrogen column density in the direction to the source. Optical magnitudes are primarily $B_{\mathrm{J}}$-magnitudes from the POSS-II plates and, as most objects lack redshift information, no K-corrections are applied. The optically identified objects are denoted by different symbols as given in the insert. Sources labelled "No ID" include those identified with faint sources classified as unresolved by COSMOS or the APM. The

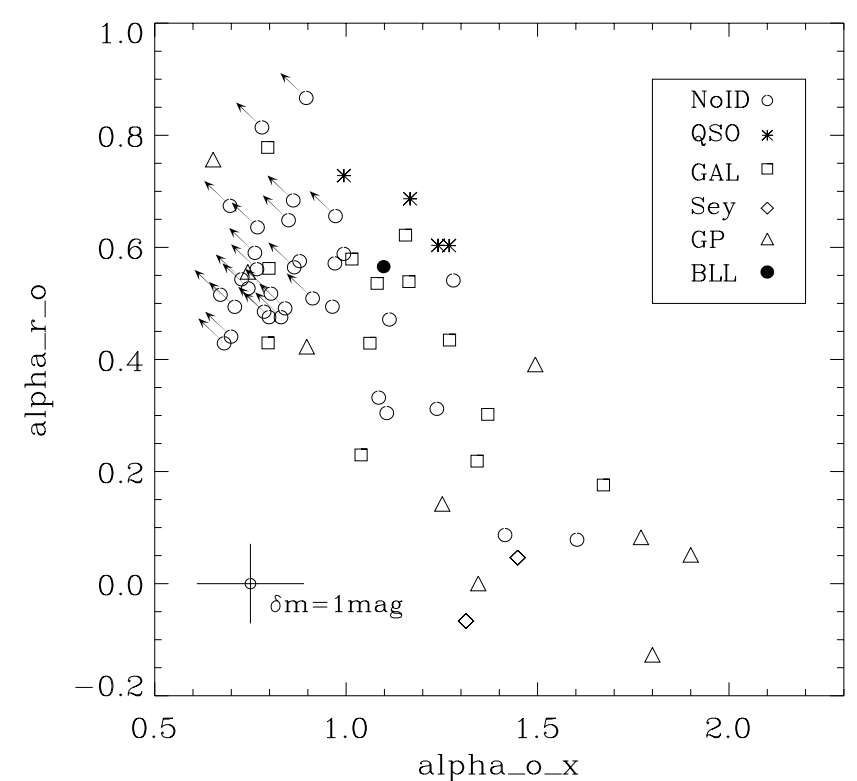

Fig. 8. Broad band energy distribution of all sources. The axes are the radio-to-optical and optical-to-X-ray spectral indices as defined in the text. Arrows indicate objects with upper limits in the optical magnitude

cross indicates the ranges of the indices corresponding to an optical error of $\delta m= \pm 1$ magnitude.

The bulk of the objects are found along the diagonal swath from high- $\alpha_{\text {ro }}$ and low- $\alpha_{\text {ox }}$ to low- $\alpha_{\text {ro }}$ and high- $\alpha_{\text {ox }}$. This swath is the region generally occupied by radio-loud quasars and blazars (see Fig. 9 in Paper I). Only four of the objects in Tables 1 and 2 are spectroscopically identified as quasars and one is the well known BL Lac object S5 $1749+70$. We therefore predict that most of the remaining objects are quasars or related species such as flat spectrum radio sources (upper left of the $\alpha_{\text {ro }}-\alpha_{\text {ox }}$ diagram), BL Lac objects (throughout most of the swath; LaurentMuehleisen et al. 1998), and Seyfert galaxies (lower right of the $\alpha_{\text {ro }}-\alpha_{\text {ox }}$ diagram).

A considerable fraction, $\approx 35 \%$, of the RASS-VLA sources do not have optical counterparts brighter than $B=22.5$. These are indicated by circles with arrows pointed toward the upper left of the $\alpha_{\text {ro }}-\alpha_{\text {ox }}$ diagram. Many of these have X-ray fluxes above $110^{-13} \mathrm{erg} \mathrm{s}^{-1}$ $\mathrm{cm}^{-2}$ and thus do not represent the large population of extremely faint sources thought to constitute a significant fraction of the X-ray background radiation. Those that lie in the middle or lower-right portion of the diagram are likely to be normal quasars. But those lying in the upper left portion have unusual spectral energy distributions with $\alpha_{\mathrm{ro}} \simeq \alpha_{\mathrm{ox}}$ or even inverted spectra with $\alpha_{\text {ro }}>\alpha_{\text {ox }}$. These sources can arise in three plausible ways: (a) they are distant clusters whose X-ray emission is elevated due to an intra cluster medium; (b) they are members of a subclass of optically-quiet quasars or red quasars (e.g. Webster et al. 1995; Kollgaard et al. 1995); or (c) they 
are spurious X-ray/radio correlations. Option (c) is likely correct for those sources with very weak radio emission, but over half (11 out of 21, see Fig. 11) have $S_{1.5}>5 \mathrm{mJy}$ and almost a third have $S_{1.5}>10 \mathrm{mJy}$, for which spurious coincidences are unlikely. It is unclear whether the quasars in option (b) constitute a rare or a major class of AGN. We looked at the three most radio-bright NEP examples in a search for optically-quiet quasars (Kollgaard et al. 1995) and concluded that they were more or less typical radio galaxies.

The source $1747.2+6532$ in the upper left in Fig. 8 at $\alpha_{\text {ro }} \sim 0.78$ is identified as $4 \mathrm{C}+65.22$. Lacy et al. (1993) mark it as "Q?" based on CCD observations. Kollgaard et al. (1995) argue that $4 \mathrm{C}+65.22$ is not a quasar, but a radio galaxy, based on the two-component radio morphology at $8.4 \mathrm{GHz}$, associated spectral indices, and marginallyresolved optical ( $R=21.2 \mathrm{mag})$ image. It is undetected on the blue POSS II plate. However, its position in the diagram, and even more that in the flux-ratio plot (Fig. 10) where it is found close to left boundary, at $\log \left(f_{\mathrm{x}} / f_{\mathrm{r}}\right)$ $\sim-6.7$, would be very unusual for a galaxy and thus the object may be a quasar instead. The RASS-VLA source $1743.4+6342$ which we classify as a group/cluster occupies an extreme position in the diagram as well, and it may be spurious in some sense. The radio source, also known as 8C $1743+637$, arises from a faint galaxy behind the cluster Abell 2280 (Lacy et al. 1993), while the X-ray emission is likely due to the Abell cluster's gaseous medium. In any case, the extended X-ray RASS source represents the detection of a galaxy grouping.

The lower right region of the diagram, which is usually populated by optically prominent galaxies with low X-ray and radio emission, remains relatively empty. We suggest that another order of magnitude in X-ray sensitivity is needed before normal spiral galaxies are commonly found in all-sky surveys. Instead, we find optical counterparts that are morphologically classified as galaxies all along the swath occupied in the $\alpha_{\text {ro }}-\alpha_{\text {ox }}$ diagram. This is equivalent to the statement that galaxy magnitudes are roughly uniformly distributed over the range $14 \leq B \leq 21$. These objects include two IRAS-selected Seyfert 1 galaxies at $z \simeq 0.05-0.08$, a nearby Seyfert 2 galaxy at $z \simeq 0.02$, and several distant unstudied (presumably elliptical) radio galaxies. In the latter cases, the X-ray emission may arise either from the active nucleus or from a surrounding intracluster medium.

Finally, we note that the galaxy groups and clusters are spread throughout the diagram, illustrating the lack of tight correlations between optical, radio and X-ray luminosities from these objects (Burns et al. 1994). The $\alpha_{\text {ro }}-\alpha_{\text {ox }}$ diagram is thus a poor diagnostic tool for discriminating groups and clusters from AGN.

\subsection{The $\alpha_{\text {ro }}$ distribution and the radio-loud criterion}

We use the radio-to-optical spectral index, $\alpha_{\text {ro }}$, to analyze the NEP sample for evidence of the apparent dichotomy between radio-quiet and radio-loud AGN. Both $\alpha_{\text {ro }}$ and its equivalent, the ratio of radio to optical flux densities (or luminosities), $R$, have been extensively used for this purpose. The commonly accepted dividing line between the two populations, established by studies of opticallyselected AGN, is $\log R \sim 1$ (Kellerman et al. 1989; Stocke et al. 1992), or $\alpha_{\text {ro }} \sim 0.2$. The flux densities are typically taken at $5 \mathrm{GHz}$ and an observed wavelength of $4400 \AA$, taken from the $B$ magnitude. A second criterion is the radio luminosity; the division occurs $\sim L_{5} \mathrm{GHz}=10^{24}$ $10^{26}\left[\mathrm{~W} \mathrm{~Hz}^{-1}\right]$ (Miller et al. 1990; Stocke et al. 1992), with some dependence on redshift (Padovani 1993). Miller et al. (1990) note that the luminosity criterion is to be preferred if the optical and radio emission of AGN are not correlated. The two definitions overlap by approximately 90\% (Kellerman et al. 1994; Della Ceca et al. 1994). Falcke et al. (1996) have proposed separate radio-loud - radioquiet criteria for flat- and steep-spectrum quasars: $R \simeq$ 25 ( $\left.\alpha_{\text {ro }} \simeq 0.24\right)$ for steep-spectrum sources and $R \sim 250$ $\left(\alpha_{\text {ro }} \sim 0.44\right)$ for flat-spectrum.

The radio-loud - radio-quiet dichotomy is most evident as bimodality in the distributions of the measures $R, \alpha_{\mathrm{ro}}$, or $L_{\mathrm{R}}$. Bimodality has been reported for both optically-selected samples (e.g. Sramek \& Weedman 1980; Kellerman et al. 1989, 1994; Miller et al. 1990; Visnovsky et al. 1992; Stocke et al. 1992) and the X-ray-selected sample of AGN from the Einstein Medium Sensitivity Survey (EMSS; Della Ceca et al. 1994). Bimodality is sometimes evident in the distribution of radio flux densities, at least for optically-selected samples (Strittmatter et al. 1980; Kellerman et al. 1989). Evidence in terms of other observed AGN properties, such as redshift and optical luminosity, has been more difficult to establish.

Figure 9 shows the distribution of $\alpha_{\text {ro }}$ values for the reliable NEP sources (Tables 1 and 2). A bimodal distribution is strongly suggested, with a minimum $\sim 0.2$. This is in good agreement with the minimum value of $\alpha_{\text {ro }}$ found for optically-selected samples and the value of $\alpha_{\text {ro }}=0.35$ obtained by Della Ceca et al.'s results for EMSS AGN. (The different radio frequencies and the lack of K-corrections for the NEP sample would change $\alpha_{\text {ro }}$ by approximately \pm 0.05 .) It should be noted that the NEP sources include non-AGN (such as the apparent $\mathrm{cD}$ galaxies of several group candidates). As the NEP and EMSS samples have roughly similar sensitivities in the radio ( $\sim 1 \mathrm{mJy}$ at 1.5 and $5 \mathrm{GHz}$, respectively), optical $(\sim 23 \mathrm{mag}$ in $V$ and $B)$, and X-ray $\left(\gtrsim 510^{-14} \mathrm{erg} \mathrm{s}^{-1}\right.$ for $0.1-2.4 \mathrm{keV}$ and $\gtrsim 810^{-14} \mathrm{erg} \mathrm{s}^{-1}$ for $\left.0.3-3.5 \mathrm{keV}\right)$, the NEP sample's results provide further confirmation of bimodality but do not extend it to significantly fainter limits. The minimum in the $\alpha_{\text {ro }}$ distribution is also evident as a sparsely-populated band ("gap") in the $\alpha_{\text {ro }}-\alpha_{\text {ox }}$ and 


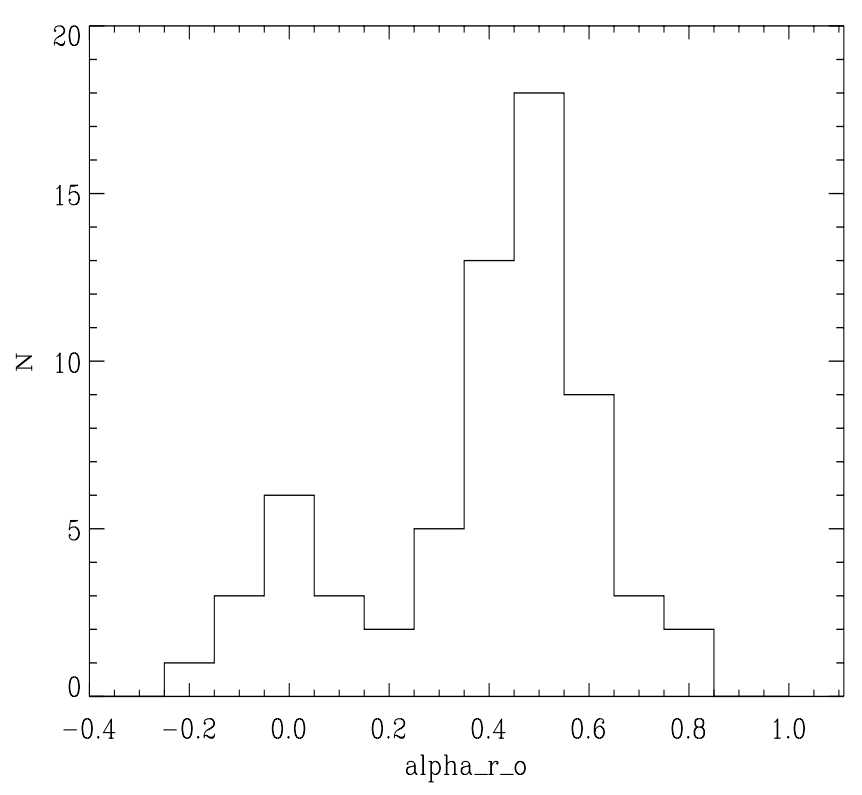

Fig. 9. Distribution of the radio-to-optical spectral index $\alpha_{\text {ro }}$. All sources in Tables 1 and 2 are plotted, including those with upper limits on their optical flux

$f_{\mathrm{x}} / f_{\mathrm{r}}-f_{\mathrm{o}} / f_{\mathrm{r}}$ (Sect. 5.4) plots. The gap is most apparent if different morphological classes are considered separately. Although bimodality is not evident in RGB sample (its $\alpha_{\text {ro }}$ distribution cuts off at the lower limit of radio-loudness), it is quite evident in the sample of radio-selected, ROSATdetected quasars drawn from the Véron-Cetty - Véron (1993) catalog (VV93) studied by Brinkmann et al. (1997; see Fig. 16).

\subsection{The $f_{x} / f_{r}-f_{o} / f_{r}$ diagram}

While AGN classes often overlay in the $\alpha_{\mathrm{ro}}-\alpha_{\mathrm{ox}}$ diagram, they can occupy more distinct regions in the $f_{\mathrm{x}} / f_{\mathrm{r}}-f_{\mathrm{o}} / f_{\mathrm{r}}$ (or equivalently, $\alpha_{\mathrm{rx}}-\alpha_{\text {ro }}$ ) diagram. For example, X-rayselected BL Lacs and radio-selected BL Lacs are widely separated in this diagram with BL Lacs from the RGB survey filling the gap between these populations (Fig. 11 in Paper II; Laurent-Muehleisen et al. 1998). Figure 10 shows the flux ratio diagram for objects in Tables 1 and 2. Objects on the top right should be mainly galaxies, $\mathrm{X}$-ray-selected $\mathrm{BL}$ Lacs reside in the top central region, while quasars and radio-selected BL Lac objects congregate at the lower left. The diagram is similar to that of the optically unidentified objects from the RGB survey (Fig. 11 in Paper II), indicating that the populations have not changed dramatically between the brighter RGB and fainter NEP samples. The optically faint and unclassified NEP sources are spread throughout the region of radioand X-ray-selected AGN. However, few are present among the Seyferts and other galaxies in the radio-quiet regime, $\log \left[f_{\mathrm{o}} / f_{\mathrm{r}}\right]>-1(R \lesssim 10)$. The $f_{\mathrm{x}} / f_{\mathrm{r}}-f_{\mathrm{o}} / f_{\mathrm{r}}$ diagram is

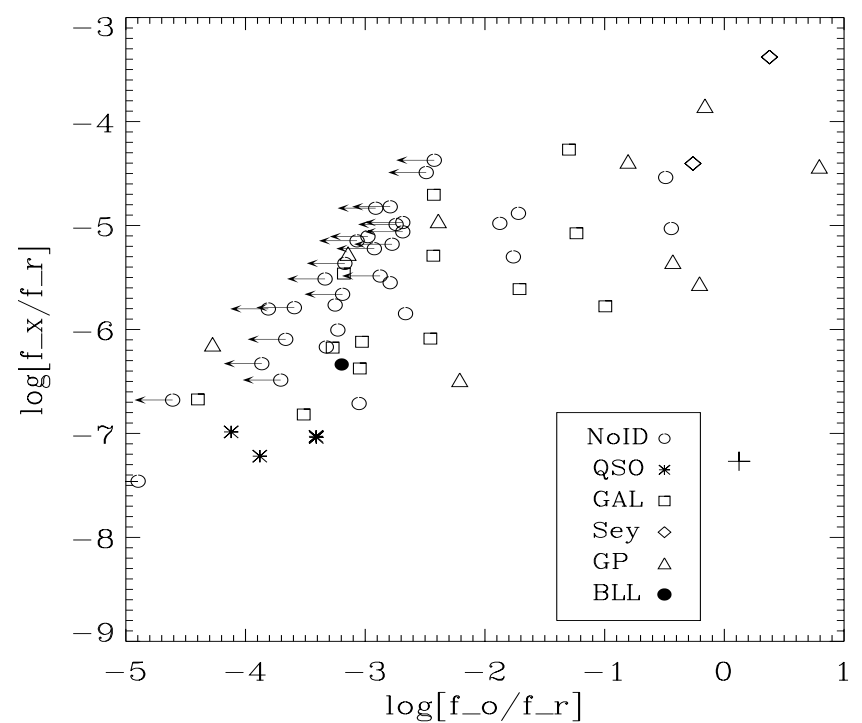

Fig. 10. Flux ratios $\log \left[f_{\mathrm{x}} / f_{\mathrm{r}}\right]$ versus $\log \left[f_{\mathrm{o}} / f_{\mathrm{r}}\right]$ for all sources. Arrows indicate sources with upper limits for the optical fluxes. The cross marks the position of the planetary nebula

consistent with Fig. 8 in suggesting that several source identifications may be incorrect.

\section{Summary}

We have presented an unbiased survey of extragalactic objects chosen by their simultaneous presence in flux-limited $\mathrm{X}$-ray and radio continuum surveys. While other studies have penetrated deeper over small fields or surveyed larger areas with lower sensitivity, our effort is unique in its combination of depth (reaching $510^{-14} \mathrm{erg} \mathrm{s}^{-1} \mathrm{~cm}^{-2}$ in the $0.1-2.4 \mathrm{keV}$ band and $1 \mathrm{mJy}$ at $1.5 \mathrm{GHz}$ ) and areal extent (29.3 square degrees). It was made possible by the repeated scans of the North Ecliptic Pole region during the ROSAT All-Sky Survey and a complementary radio survey with the VLA.

A total of 74 reliable RASS-VLA sources were found (including multiple radio components), with an additional 34 possible matches. Optical counterparts were sought on digitized Schmidt plates of the POSS-I and II surveys. The result is a rather heterogeneous sample with objects ranging in brightness by 2 orders of magnitude in the $\mathrm{X}$-rays, 3 orders of magnitude in the radio and $>3$ orders of magnitude in the optical. The minority of objects with optical spectroscopy include four quasars, one BL Lac object, two Seyfert 1 galaxies, and two Seyfert 2 galaxies. Unstudied objects include galaxies with $15 \leq B \leq 22$ and unresolved objects which are presumably quasars and BL Lacs. A considerable fraction (approximately one-third) of the radio- and X-ray-loud objects do not have any optical counterparts brighter than $B=22.5$.

The previously identified objects do not show any class-specific separation with regard to their radio or 


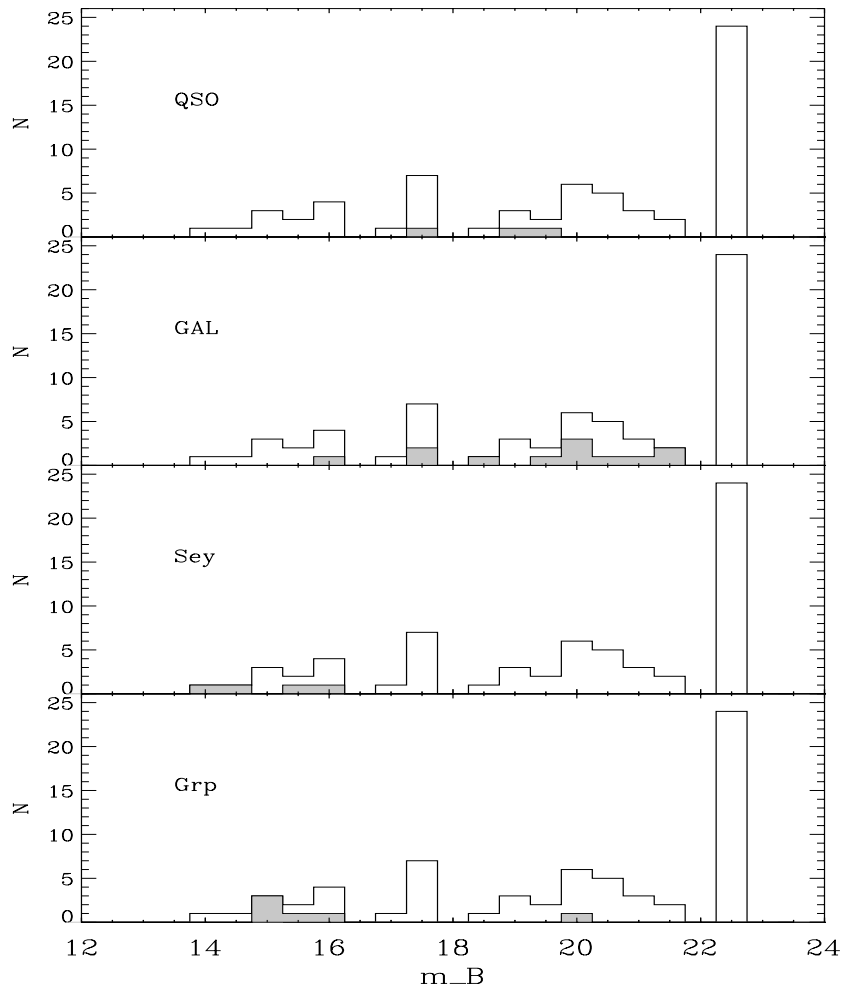

Fig. 11. Distribution of various object types as function of the optical $B$ - magnitude. Open histogram: all objects; shaded region: specific object class

X-ray fluxes. Only the optical magnitudes indicate some systematic trend for the various objects (see Fig. 11). For 23 sources only upper limits could be obtained $\left(m_{B}>22.5\right.$; one source was detected on a red plate). However, seven of these are quite strong radio sources with fluxes $f_{\mathrm{r}}>$ $10 \mathrm{mJy}$.

While the heterogeneity of the sample and incompleteness of redshift information precludes quantitative analysis, a number of interesting findings emerge. They mainly support similar results that emerged from the shallower but large-area RGB survey discussed in Papers I, II and Laurent-Muehleisen et al. (1998).

1. The distribution of radio-to-optical flux ratios and spectral indices appears to be bimodal, in agreement with optically-selected and other X-ray/radio-selected samples.

2. Ten likely galaxy groups and clusters are detected, including two Abell clusters and four groups found in previous X-ray surveys with optical (but not radio) follow-up. The X-ray/radio detection strategy appears to be quite effective in uncovering relatively poor clusters which contain a radio galaxy.

3. Many of the RASS-VLA sources are optically very faint for their radio and X-ray fluxes compared to normal quasars with $\alpha_{\text {ro }} \simeq \alpha_{\text {ox }}$ or even $\alpha_{\text {ro }}>\alpha_{\text {ox }}$. These are either "red quasars" or new distant clusters containing a radio galaxy.

Acknowledgements. The ROSAT project is supported by the Bundesministerium für Bildung, Wissenschaft, Forschung und Technologie (BMBF/DLR) and the Max-Planck-Gesellschaft. We thank Peter Kahabka for his help in programming the source detection routines and our colleagues from the ROSAT group for their support. This research at Penn State was supported by NASA grant NAGW-2120 to EDF. RIK acknowledges support from Fermi National Accelerator Laboratory; MMC acknowledges partial support from Bucknell University. We acknowledge the assistance of W.L.W. Sargent (CalTech) in making POSS-II plates of the NEP available to ROE for Cosmos digital scanning and thank Richard McMahon for providing the APM data. This research has made use of the NASA/IPAC Extragalactic Data Base (NED) which is operated by the Jet Propulsion Laboratory, California Institute of Technology, under contract with the National Aeronautics and Space Administration.

\section{References}

Abell G.O., Corwin Jr. H.G., Olowin R.P., 1989, ApJS 70, 1 Aschenbach B., 1988, Appl. Opt. 27, 1404 Ashby M.L.N., Hacking P.B., Houck J.R., Soifer B.T., Weisstein E.W., 1996, ApJ 456, 428

Becker R.H., White R.L., Helfand D.J., 1995, ApJ 450, 559

Benn C.R., Rowan-Robinson M., McMahon R.G., Broadhurst T.J., Lawrence A., 1993, MNRAS 263, 98

Bower R.G., Hasinger G., Castander F.J., et al., 1996, MNRAS 281, 59

Boyle B.J., Fong G.R., Shanks T., Peterson B.A., 1990, MNRAS 243, 1

Boyle B.J., Griffiths R.E., Shanks T., et al., 1993, MNRAS 260,49

Brinkmann, W., 1994, in: Courvoisier T.J.-L., Blecha A. (eds.), Multi-Wavelength Continuum Emission of AGN. Kluwer Academic Publishers, Dordrecht, p. 53

Brinkmann W., Siebert J., Boller Th., 1994, A\&A 281, 355

Brinkmann W., Siebert J., Reich W., et al., 1995, A\&AS 109, 147 (Paper I)

Brinkmann W., Siebert J., Feigelson E.D., et al., 1997, A\&A 323, 739 (Paper II)

Brinkmann W., Yuan W., Siebert J., 1997, A\&A 319, 413

Burg R., Giacconi R., Huchra J., et al., 1992, A\&A 259, L9

Burns J.O., Rhee G., Owen F.N., Pinkney J., et al., 1994, ApJ 423, 94

Carballo R., Warwick R.S., Barcons X., et al., 1995, MNRAS 277,1312

Condon J.J., Broderick J.J., Seielstad G.A., 1989, AJ 97, 1064

Condon J.J., Cotton W.D., Greisen E.W., et al., 1998, ApJ 115,1693

Cruddace R.C., Hasinger G.H., Schmitt J.H., 1988, in Astronomy from Large Databases, Murthagh F. and Heck A. (eds.), p. 177

Della Ceca R., Zamorani G., Maccacaro T., et al., 1994, ApJ 430,533

de Ruiter, H.R., et al., 1994, in Frontiers of Space \& GroundBased Astronomy, Wamsteker W., Longair M.S. and Kondo Y. (eds.). Kluwer Academic Press, p. 673 
de Ruiter, H.R., Zamorani G., Parma P., et al., 1997, A\&A 319,7

Dickey J.M., Lockman F.J., 1990, ARA\&A 28, 215

Elvis M., Lockman F.J., Fassnacht C., 1994, APJS 95, 413

Falcke H., Sherwood W., Patnaik A.R., 1996, ApJ 471, 106

Georgantopoulos I., Stewart G.C., Shanks T., et al., 1996, MNRAS 280, 276

Gioia I.M., Maccacaro T., Schild R.E., et al., 1990, ApJS 72, 567

Gioia I.M., Luppino G.A., 1994, ApJS 94, 583

Gioia I.M., Henry, J.P., Luppino G.A., et al., 1995, A\&A 297, L75

Gómez P.L., Pinkney J., Burns J.O., 1997, ApJ 474, 580

Gregg M.D., Becker R.H., White R.C., et al., 1996, AJ 112, 497

Hacking P., Houck J.R., 1987, ApJS 63, 311

Hamilton T.T., Helfand D.J., 1993, ApJ 418, 55

Hasinger G., Schmidt M., Trümper J., 1991, A\&A 246, L2

Henry J.P., Gioia I.M., Maccacaro T., et al., 1992, ApJ 386, 408

Henry J.P., Gioia I.M., Huchra J.P., et al., 1995, ApJ 449, 422

Hertz P.L., Kollgaard R.I., Chester M.M., et al., 1994, Naval Research Lab. Report NRL/MR/7621-94-7439, Washington

Kellerman K.I., Sramek R., Schmidt M., et al., 1989, AJ 98, 1195

Kellerman K.I., Sramek R., Schmidt M., et al., 1994, AJ 108, 1163

Kock A., Meisenheimer K., Brinkmann W., et al., 1996, A\&A 307, 745

Kollgaard R.I., Brinkmann W., Chester M.M., et al., 1994, ApJS 93, 145

Kollgaard R.I., Feigelson E.D., Laurent-Muehleisen S.A., et al., 1995, ApJ 449, 61

Kreysing H.C., Diesch C., Zweigle J., et al., 1992, A\&A 264, 623

Kühr H., Nauber U., Pauliny-Toth I.I.K., Witzel A., 1979, MPIfR preprint No. 55

Lacy M., Rawlings S., Warner P.J., 1992, MNRAS 256, 404

Lacy M., Hill G.J., Kaiser M.-E., Rawlings S., 1993, MNRAS 263, 707

Lacy M., Riley J.M., Waldram E.M., McMahon R.G., Warner P.J., 1995, MNRAS 276, 614

Laurent-Muehleisen S.A., 1996, Ph.D. Thesis, The Pennsylvania State University

Laurent-Muehleisen S.A., Kollgaard R.I., Ryan P.J., et al., 1997, A\&AS 122, 235

Laurent-Muehleisen S.A., Kollgaard R.I., Ciardullo R., et al., 1998, ApJS 118 (in press)

Large M.I., Cram L.E., Burgess A.M., 1991, The Observatory 111,72

Loiseau N., Reich W., Wielebinski R., Reich P., Münch W., 1988, A\&AS 75, 67

Maccacaro T., Wolter A., McLean B., et al., 1994, Astro. Lett.
Comm. 29, 267

MacGillivray H.T., Stobie R.S., 1984, Vistas Astron. 27, 433

McMahon R.G., 1991, in: The Space Distribution of Quasars, Crampton D. (ed.), ASP Conf. Ser. 21, 129

McMahon R.G., Irwin M.J., 1992, in: Digitised Optical Sky Surveys, MacGillivray H.T., Thomson E.B. (eds.). Kluwer, Dordrecht, p. 417

Miller L., Peacock J.A., Mead A.R.G., 1990, MNRAS 244, 207

Owen F.N., Ledlow M.J., Keel W.C., 1996, AJ 111, 53

Padovani P., 1993, MNRAS 263, 461

Pfeffermann E., Briel U.G., Hippmann H., et al., 1986, Proceedings SPIE 733, 519

Pierre M., Hunstead R., Reid A., et al., 1994, The Messenger 78,24

Reid I.N., Brewer C., Brucato R.J., et al., 1991, PASP 103, 661

Rusk R., Seaquist E.R., 1985, AJ 90, 30

Sarazin C.L., 1986, in: Radio continuum processes in Clusters of Galaxies, NRAO Workshop Nr. 16, O'Dea P., Uson J.M. (eds.). Green Bank, p. 23

Sramek R.A., Weedman D.W., 1980, ApJ 238, 435

Stocke J.T., Morris S.L., Gioia I.M., et al., 1991, ApJS 76, 813

Stocke J.T., Morris S.L., Weymann R.J., Foltz C.B., 1992, ApJ 396, 487

Strittmatter P.A., Hill P., Pauliny-Toth I.I.K., et al., 1980, A\&A 88, L12

Thuan T.X., Patterson R.J., Condon J.J., Mitchell K.J., 1992, AJ 104, 1331

Trümper J., 1983, Adv. Space Res. 2, 241

Veron-Cetty M.-P., Veron P., 1993, A Catalogue of Quasars and Active Nuclei (6th Edition), ESO Scientific Report No. 13

Veron-Cetty M.-P., Veron P., 1996, A Catalogue of Quasars and Active Nuclei (7th Edition), ESO Scientific Report No. 17

Visnovsky K.L., Impey C.D., Foltz C.B., et al., 1992, ApJ 391, 560

Voges W., 1992, in: Proc. of the ISY Conference "Space Science", ESA ISY-3, ESA Publications, p. 9

Voges W., Boller Th., Dennerl K., et al., 1996, in: Proceedings of the Conference Röntgenstrahlung from the Universe, MPE Report 263, p. 637

Warwick R.S., Barber C.R., 1992, in: Proceedings of the Conference X-ray Emission from AGN and the Cosmic X-ray Background, MPE Report 235, p. 242

Webster R.L., Francis P.J., Peterson B.A., et al., 1995, Nat 375,469

Windhorst R.A., Miley G.K., Owen F.N., 1985, ApJ 289, 494

Wood K.S., Meekins J.F., Yentis D.J., et al., 1984, ApJS 56, 507

Yentis D.J., Cruddace R.G., Gursky H., et al., 1992, in: Digitised Optical Sky Surveys, MacGillivray H.T., Thomson E.B. (eds.). Kluwer, Dordrecht, p. 67

Zimmermann H.U., Becker W., Belloni T., et al., 1994, EXSAS User's Guide, Edt. 4, MPE Report 257 
Table 1. Compact X-ray/radio sources in the NEP

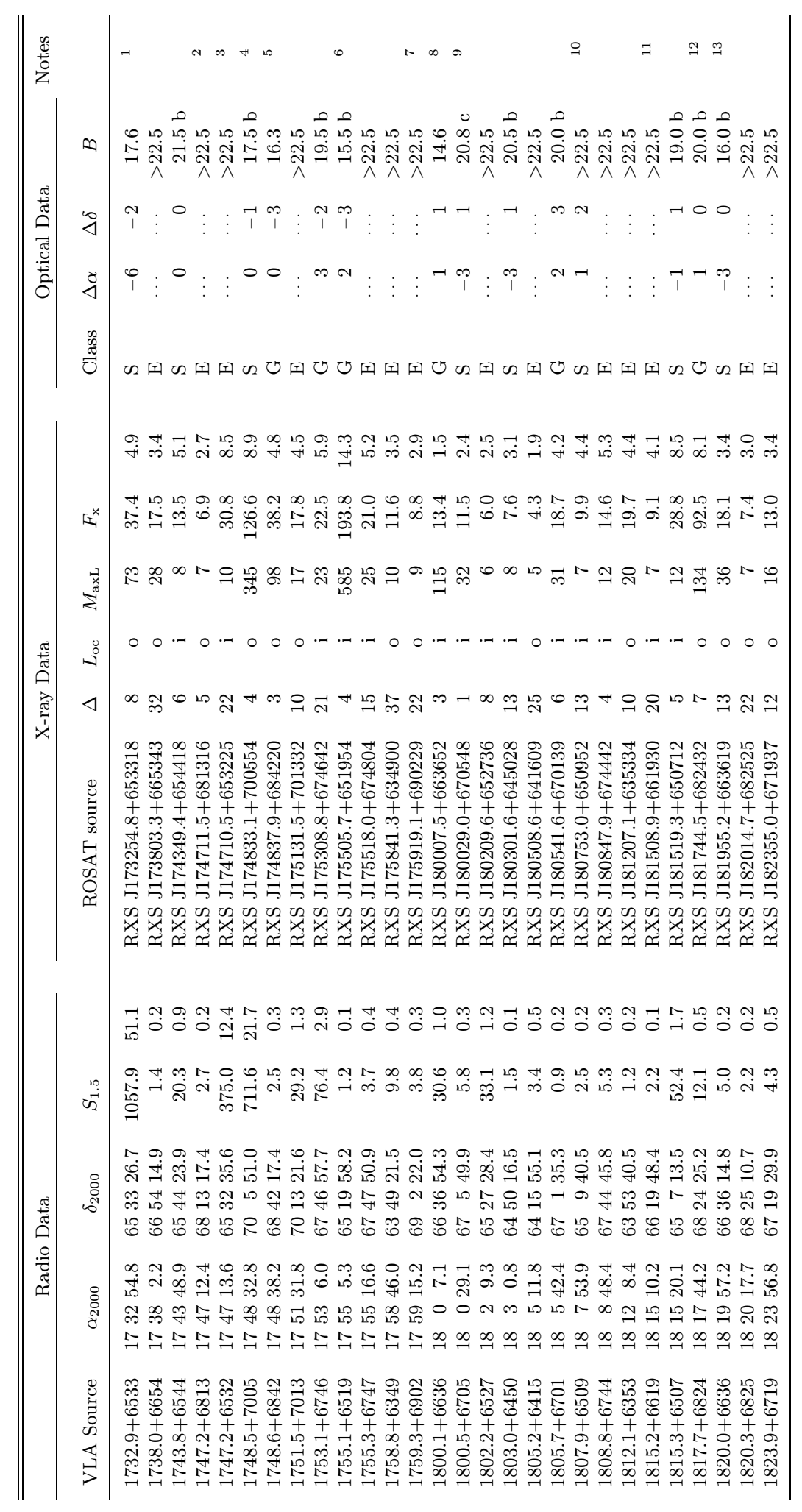

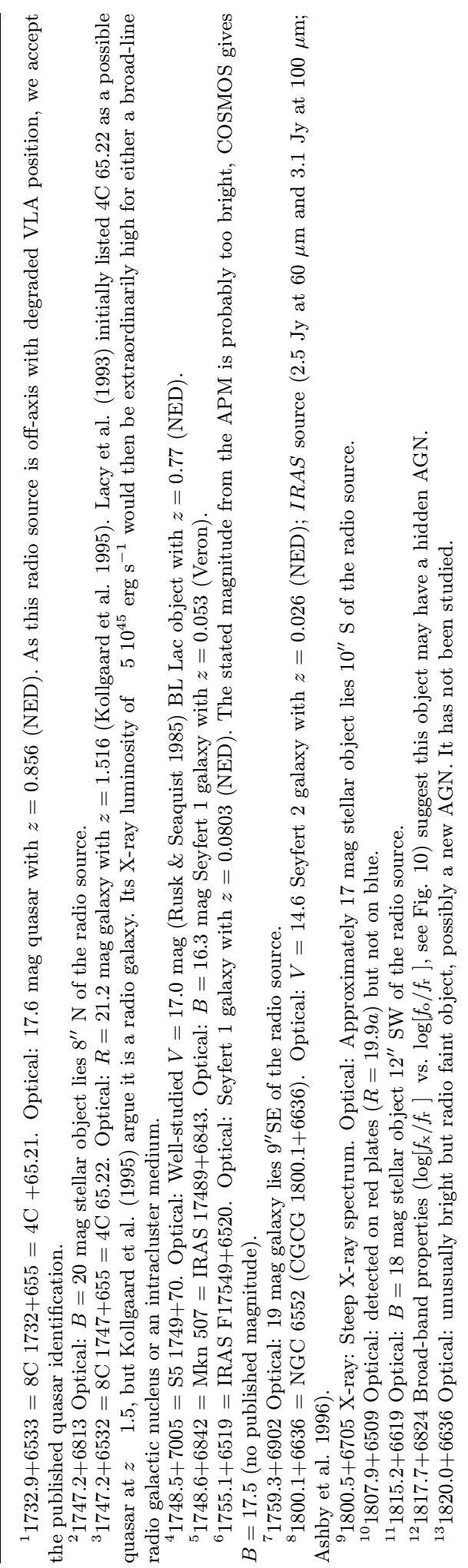




\begin{tabular}{|c|c|c|c|c|c|c|c|c|c|c|c|c|c|c|c|}
\hline \multicolumn{5}{|c|}{ Radio Data } & \multicolumn{6}{|c|}{ X-ray Data } & \multicolumn{4}{|c|}{ Optical Data } & \multirow[t]{2}{*}{ Notes } \\
\hline VLA Source & $\alpha_{2000}$ & $\delta_{2000}$ & $S_{1.5}$ & & ROSAT source & $\Delta$ & $L_{\mathrm{oc}}$ & $M_{\mathrm{axL}}$ & $F_{\mathrm{x}}$ & & Class & $\Delta \alpha$ & $\Delta \delta$ & $B$ & \\
\hline $1741.3+6508$ & 174116.0 & $\begin{array}{lll}65 & 8 & 6.0\end{array}$ & 2.8 & 0.3 & RXS J174114.3+650748 & 21 & o & 20 & 16.4 & 4.0 & $\mathrm{E}$ & & $\cdots$ & $>22.5$ & 1 \\
\hline $1743.1+6732$ & $1743 \quad 7.8$ & 673232.5 & 17.8 & 0.6 & RXS J174312.1+673220 & 27 & $\mathrm{i}$ & 7 & 11.5 & 4.8 & $\mathrm{G}$ & 6 & -1 & $16.0 \mathrm{c}$ & 2 \\
\hline $1743.4+6342$ & 174323.5 & 634257.8 & 214.4 & 9.5 & RXS J174328.0+634145 & 78 & o & 69 & 59.8 & 7.0 & $\mathrm{Gp}$ & & $\cdots$ & 21.5 & 3 \\
\hline $1744.7+6750$ & 174442.9 & 675047.1 & 38.1 & 1.5 & RXS J174444.3+675035 & 14 & $\mathrm{i}$ & 5 & 10.0 & 4.9 & $\mathrm{~S}$ & -4 & -1 & $21.0 \mathrm{a}$ & 4 \\
\hline $1746.1+6421$ & $1746 \quad 6.4$ & 642149.5 & 237.4 & 7.4 & RXS J174605.0+642150 & 9 & o & 10 & 9.5 & 3.1 & S & 1 & 0 & $21.0 \mathrm{~b}$ & 5 \\
\hline $1747.3+6701$ & 174720.7 & $67 \quad 123.5$ & 48.4 & 1.5 & RXS J174721.6+670211 & 48 & $\mathrm{i}$ & 28 & 18.5 & 4.7 & S & 5 & 40 & $20.5 \mathrm{c}$ & 6 \\
\hline $1747.4+6702$ & 174722.8 & $\begin{array}{lll}67 & 231.0\end{array}$ & & $\cdots$ & RXS J174721.6+670211 & 21 & $\mathrm{i}$ & 28 & 18.5 & 4.7 & & $\cdots$ & $\cdots$ & $\cdots$ & 6 \\
\hline $1747.5+6345$ & 174730.9 & 634528.1 & 77.3 & 3.2 & RXS J174734.0+634511 & 27 & o & 10 & 20.0 & 5.1 & G & -3 & -3 & $20.0 \mathrm{~b}$ & 7 \\
\hline $1748.4+6404$ & 174823.0 & $64 \quad 4 \quad 5.6$ & 1.4 & 0.1 & RXS J174822.8+640340 & 25 & o & 17 & 15.7 & 3.8 & $\mathrm{~S}$ & 1 & 2 & $17.5 \mathrm{~b}$ & 8 \\
\hline $1748.5+6404$ & 174827.6 & $\begin{array}{lll}64 & 4 & 5.8\end{array}$ & $\cdots$ & $\cdots$ & RXS J174822.8+640340 & 40 & o & 17 & 15.7 & 3.8 & $\ldots$ & $\ldots$ & $\ldots$ & $\cdots$ & 8 \\
\hline $1749.4+6631$ & 174921.5 & 663152.4 & 6.7 & 0.2 & RXS J174918.3+663146 & 20 & $\mathrm{i}$ & 16 & 11.2 & 3.4 & $\mathrm{E}$ & $\ldots$ & $\ldots$ & $>22.5$ & 9 \\
\hline $1749.8+6824$ & 174948.1 & $6824 \quad 1.4$ & $\cdots$ & $\cdots$ & RXS J174948.1+682325 & 36 & o & 8 & 7.8 & 2.8 & $\ldots$ & $\cdots$ & $\cdots$ & $\ldots$ & 10 \\
\hline $1749.8+6823$ & 174950.5 & 682311.0 & 7.7 & 0.3 & RXS J174948.1+682325 & 19 & o & 8 & 7.8 & 2.8 & $\mathrm{Gp}$ & $\cdots$ & $\ldots$ & 16. & 10 \\
\hline $1751.1+6531$ & $1751 \quad 6.6$ & 653152.5 & 0.6 & 0.1 & RXS J175107.7+653204 & 13 & $\mathrm{i}$ & 7 & 8.6 & 1.7 & $\mathrm{Gp}$ & $\ldots$ & $\ldots$ & 15.4 & 11 \\
\hline $1751.4+6719$ & 175125.9 & 671927.8 & 2.4 & 0.2 & RXS J175130.2+671910 & 31 & $\mathrm{i}$ & 13 & 38.4 & 7.7 & $\mathrm{Gp}$ & 9 & 5 & $17.7 \mathrm{c}$ & 12 \\
\hline $1751.6+6719$ & 175135.2 & 671943.7 & $\cdots$ & $\cdots$ & RXS J175130.2+671910 & 45 & $\mathrm{i}$ & 13 & 38.4 & 7.7 & $\cdots$ & $\ldots$ & $\cdots$ & $\cdots$ & 12 \\
\hline $1751.9+6454 \mathrm{~A}$ & 175151.8 & 645450.4 & $\ldots$ & $\ldots$ & RXS J175151.3+645511 & 21 & $\mathrm{i}$ & 5 & 9.6 & 7.2 & $\ldots$ & $\ldots$ & $\ldots$ & $\ldots$ & 13 \\
\hline $1751.9+6454 \mathrm{~B}$ & 175153.6 & 645418.5 & 129.7 & 4.1 & RXS J175151.3+645511 & 55 & $\mathrm{i}$ & 5 & 9.6 & 7.2 & S & 4 & 0 & $19.0 \mathrm{a}$ & 13 \\
\hline $1752.0+6551$ & 175158.8 & 655123.4 & 36.9 & 1.2 & RXS J175157.3+655128 & 10 & $\mathrm{i}$ & 57 & 34.9 & 6.5 & $\mathrm{G}$ & $\ldots$ & $\ldots$ & 17.0 & 14 \\
\hline
\end{tabular}

1741.3+6508 Radio: possible triple, $24^{\prime \prime}$ separation

${ }^{2} 1743.1+6732$ Radio: extended about $30^{\prime \prime}$, possibly with strong core. Optical: Uncatalogued 16 mag galaxy, possibly elliptical with companion; dozen galaxies $>18$ mag within $10^{\prime}$.

${ }^{3} 1743.4+6342=8 \mathrm{C} 1743+637$. X-ray: extended $\left(\mathrm{ML}_{\mathrm{ext}}=16.8\right)$. Radio: extended, $30^{\prime \prime}$; located in the outer region of the extended X-ray structure. Optical: 21.5 mag galaxy in the region of Abell 2280 ( $z=0.326$, Gioia et al. 1995). However, Lacy et al. (1993) suggest this galaxy is at much higher redshift. Abell 2280 has Richness Class 0, Bautz-Morgan Class II-III, and Distance Class 6 (Abell et al. 1989). Cluster of very faint galaxies seen on POSS I (Fig. 5a)

${ }^{4} 1744.7+6750$ Radio: extended, $30^{\prime \prime}$.

$1746.1+6421=8 \mathrm{C} 1745+644$. Radio: extended, $24^{\prime \prime}$. Optical: stellar object $B=21.5 \mathrm{a}(-4.0,-0.7) .21 .9$ mag quasar with $z=1.228$ (NED).

${ }^{6} 1747.3+6701$ and $1747.4+6702$ Radio: double, 66 "separation. Optical: $B=20.5$ stellar object coincides with the X-ray position; both lie midway between the radio components.

${ }^{7} 1747.5+6345$ Radio: possibly extended, $24^{\prime \prime}$

${ }^{8} 1748.4+6404$ and $1748.5+6404$ Radio: probably double, $28^{\prime \prime}$. Optical: possibly merged stellar objects. COSMOS magnitude is $B=18.9$.

${ }^{9} 1749.4+6631$ Radio: double, 36 "separation, or core of a core-dominated triple, 95 arcsecond extent oriented SE-NW. Optical: $B \quad 19$ optical objects $244^{\prime \prime}$ away from the core to the south and west.

$1749.8+6823$ Radio: possible double, $54^{\prime \prime}$ separation, and 13 mJy compact radio source $(1749.9+6824)$ to the NE. Optical: Galaxy group. The NE radio source is associated with CGCG $1750.1+6825,15.2 \mathrm{mag}$ with $z=0.05122$ (NED). $1749.8+6824$ is associated with MCG $+11-22-005,17$ mag. $1749.8+6823$ is associated with KUG 1750+683A, 16 mag (NED). The RASS source is from the outer region where extent measurements are not accurate. (Fig. 5b).

${ }^{11} 1751.1+6531=$ NGC 6505 (CGCG 1751.1+6533). X-ray: extended $\left(\mathrm{ML}_{\mathrm{ext}}=16.3\right)$. Optical: E/SO galaxy with $B=15.4, z=0.0389(\mathrm{NED})$ with galaxy group (Henry et al. 1995; Fig. 5c).

${ }^{12} 1751.4+6719$ and $1751.6+6719$ X-ray: extended $\left(\mathrm{ML}_{\text {ext }}=15.1\right)$. Radio: triple structure; $1751.4+6719$ is the apparent core, with lobes $1751.6+6719$ and $1751.2+6719$ (Kollgaard et al. 1994). Optical: Galaxy group. The central radio source is associated with two $B=17.7 \mathrm{c}$ galaxies. COSMOS classifies the nearby 18 mag objects as stellar, but locates many objects near the plate limit. (Fig. 5d).

${ }^{13} 1751.9+6454 \mathrm{~A}$ and $1751.9+6454 \mathrm{~B}=8 \mathrm{C} 1751+649$. Radio: double, $36^{\prime \prime}$ separation. Optical: The 19.0 a mag stellar object lies between the radio sources.

${ }^{14} 1752.0+6551=7 \mathrm{C} 1751+6551$ Radio: double, $30^{\prime \prime}$ separation. Optical: 17.0 mag galaxy (NED). 


\begin{tabular}{|c|c|c|c|c|c|c|c|c|c|c|c|c|c|c|c|}
\hline \multicolumn{5}{|c|}{ Radio Data } & \multicolumn{6}{|c|}{ X-ray Data } & \multicolumn{4}{|c|}{ Optical Data } & \multirow[t]{2}{*}{ Notes } \\
\hline VLA Source & $\alpha_{2000}$ & $\delta_{2000}$ & $S_{1.5}$ & & ROSAT source & $\Delta$ & $L_{\mathrm{oc}}$ & $M_{\mathrm{axL}}$ & $F_{\mathrm{x}}$ & & Class & $\Delta \alpha$ & $\Delta \delta$ & $B$ & \\
\hline $1753.6+6634$ & 175336.4 & 663437.1 & 357.2 & 15.6 & RXS J175334.9+663440 & 9 & $\mathrm{i}$ & 7 & 4.8 & 1.9 & $\mathrm{E}$ & $\cdots$ & $\cdots$ & $>22.5$ & 15 \\
\hline $1753.6+6542$ & 175338.5 & 654218.7 & 453.4 & 17.9 & RXS J175341.7+654251 & 38 & $\mathrm{i}$ & 38 & 10.6 & 5.2 & G & $\cdots$ & $\cdots$ & $19.7 \mathrm{~b}$ & 16 \\
\hline $1753.8+6542$ & 175345.2 & 654257.8 & $\ldots$ & $\ldots$ & RXS J175341.7+654251 & 23 & $\mathrm{i}$ & 38 & 10.6 & 5.2 & . & $\ldots$ & $\ldots$ & $\ldots$ & 16 \\
\hline $1754.1+6452$ & $1754 \quad 7.3$ & $6452 \quad 3.1$ & 147.8 & 4.9 & RXS J175405.6+645201 & 11 & $\mathrm{i}$ & 6 & 8.7 & 3.9 & G & 2 & 0 & $20.0 \mathrm{~b}$ & 17 \\
\hline $1755.4+6803$ & 175524.9 & $\begin{array}{lll}68 & 3 & 12.9\end{array}$ & 6.3 & 0.3 & RXS J175514.7+680324 & 58 & $\mathrm{i}$ & 8 & 87.7 & 13.3 & Gp & $\ldots$ & $\ldots$ & $>22.5$ & 18 \\
\hline $1755.7+6754$ & 175543.5 & 675410.8 & 1.8 & 0.1 & RXS J175546.7+675421 & 21 & $\mathrm{i}$ & 8 & 98.8 & 14.6 & Gp & -4 & -2 & 16. c & 19 \\
\hline $1756.5+6512$ & 175629.2 & 651250.1 & 7.6 & 0.4 & RXS J175632.2+651233 & 25 & $\mathrm{i}$ & 14 & 13.2 & 4.3 & $\mathrm{Gp}$ & $\ldots$ & $\ldots$ & 15.6 & 20 \\
\hline $1758.6+6637$ & 175833.5 & 663758.9 & 939.4 & 31.8 & RXS J175833.4+663758 & 1 & $\mathrm{i}$ & 169 & 19.6 & 1.8 & $\mathrm{PN}$ & $\cdots$ & $\cdots$ & 8.9 & 21 \\
\hline $1801.2+6902$ & $18 \quad 114.5$ & $69 \quad 240.4$ & 202.7 & 6.3 & RXS J180115.3+690244 & 6 & o & 8 & 7.5 & 2.7 & $\mathrm{~S}$ & 0 & 3 & 19.4 & 22 \\
\hline $1802.0+6657$ & $\begin{array}{ll}18 & 157.8\end{array}$ & $\begin{array}{lll}66 & 57 & 14.7\end{array}$ & 23.0 & 0.7 & RXS J180155.5+665540 & 95 & $\mathrm{i}$ & 11 & 2.9 & 0.9 & $\mathrm{E}$ & . & $\cdots$ & $>22.5$ & 23 \\
\hline $1803.9+6548$ & $18 \quad 354.0$ & 654825.0 & 43.1 & 1.7 & RXS J180353.9+654827 & 3 & $\mathrm{i}$ & 152 & 57.5 & 6.6 & $\mathrm{G}$ & 1 & 0 & $20.5 \mathrm{~b}$ & 24 \\
\hline $1805.3+6621$ & $\begin{array}{lll}18 & 5 & 16.3\end{array}$ & $\begin{array}{lll}66 & 21 & 9.8\end{array}$ & 7.0 & 0.3 & RXS J180515.8+662053 & 16 & $\mathrm{i}$ & 6 & 5.9 & 2.2 & $\mathrm{E}$ & . & $\cdots$ & $>22.5$ & 25 \\
\hline $1805.5+6535$ & $18 \quad 530.5$ & 653522.7 & 3.4 & 0.1 & RXS J180532.5+653521 & 13 & $\mathrm{i}$ & 7 & 13.8 & 4.8 & $\mathrm{~S}$ & 5 & -3 & $20.0 \mathrm{c}$ & 26 \\
\hline $1806.8+6536$ & $18 \quad 6 \quad 46.4$ & 653637.1 & 11.1 & 0.4 & RXS J180651.6+653740 & 71 & $\mathrm{i}$ & 34 & 47.5 & 8.3 & Gp & 5 & 2 & $20.0 \mathrm{c}$ & 27 \\
\hline $1808.1+6605$ & $\begin{array}{lll}18 & 8 & 3.8\end{array}$ & $\begin{array}{lll}66 & 5 & 33.3\end{array}$ & 17.7 & 0.6 & RXS J180803.4+660531 & 3 & $\mathrm{i}$ & 17 & 11.1 & 3.2 & $\mathrm{E}$ & 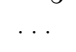 & & $>22.5$ & 28 \\
\hline
\end{tabular}

${ }^{15} 1753.6+6634=7 \mathrm{C} 1753+6635$ Radio: possible double with $1753.6+6635,72$ " separation. Optical: Two $B=18.0 \mathrm{c}$ stellar objects lie within the radio structure, but identification is unclear.

${ }^{16} 1753.6+6542$ and $1753.8+6542=8 \mathrm{C} 1753+657$. Radio: double, $54^{\prime \prime}$ separation. Optical: $B=19.9, R=17.4$ mag quasar with $z=0.148$ (Lacy et al. $1995 ;$ Kock et al. 1996). Quasar and RASS source lie between the radio peaks.

${ }^{17} 1754.1+6452$ Radio: possibly extended, $36^{\prime \prime}$.

${ }^{18} 1755.4+6803$ X-ray: Gioia \& Luppino (1994) note slightly extended X-ray emission from the EMSS cluster MS1754.9+6803 $(z=0.077)$, but it is uncertain whether the RASS-VLA source is physically related to the EMSS cluster. Radio: double, 18"separation. Optical: Galaxy group. (Fig. 5e).

${ }^{19} 1755.7+6754$ X-ray: extended $\left(\mathrm{ML}_{\text {ext }}=31.0\right)$. Radio: faint. Optical: Erroneously classified by COSMOS as a 16.4 mag star surrounded by a cluster of 21 mag objects. POSS I image indicates an elliptical galaxy with a diffuse halo ' $40^{\prime \prime}$ in extent. Possible cD galaxy in cluster. (Fig. 5f).

${ }^{20} 1756.5+6512=$ CGCG $1756.3+6513$. X-ray: extended $\left(\mathrm{ML}_{\text {ext }}=26.5\right)$. Radio: coincides with bright spot in complex X-ray structure. Optical: $B=15.6 \mathrm{E} / \mathrm{S} 0$ galaxy with $z=0.0263$ (NED) in group (Henry et al. 1995). (Fig. 5g).

${ }^{21} 1758.6+6637=$ NGC $6543=$ IRAS $17584+6638$. X-ray: Kreysing et al. 1992 found extended X-ray emission. Radio: extended, $36^{\prime \prime}$. Optical: Galactic planetary nebula.

${ }^{22} 1801.2+6902=8 \mathrm{C} 1801+690$. Radio: extended, $24^{\prime \prime}$. Optical: 19.4 mag quasar with $z=1.271$ (NED)

${ }^{23} 1802.0+6657$ Radio: double with $1802.1+6657$, separation $54^{\prime \prime}$ with marginally resolved core. Optical: No object found near core. Contour map in Kollgaard et al. $(1994)$.

${ }^{24} 1803.9+6548 \mathrm{X}$-ray: possibly extended $\left(\mathrm{ML}_{\text {ext }}=6.8\right)$. Radio: possibly extended, $36^{\prime \prime}$. The low likelihood of X-ray extension and precisely coincident radio and X-ray centroids suggest it is an unresolved active nucleus rather than a group candidate.

${ }^{25} 1805.3+6621$ Radio: possibly extended, $30^{\prime \prime}$. Optical: $17.5 \mathrm{c}$ mag stellar object located $12^{\prime \prime} \mathrm{NW}$ of the radio source.

${ }^{26} 1805.5+6535$ Radio: double or triple, $30^{\prime \prime}$ separation. Contour map in Kollgaard et al. (1994). Optical: $B=20.0$ c stellar object coincident with brightest radio component. Several other faint objects lie within $1^{\prime}$.

${ }^{27} 1806.8+6536$ X-ray: extended $\left(\mathrm{ML}_{\mathrm{ext}}=10.8\right)$. Radio: unresolved. Optical: Several $19-20$ mag objects lie within 1'. Possible distant galaxy group. (Fig. 5i).

${ }^{28} 1808.1+6605=7 \mathrm{C} 1807+6604$. Radio: double, $24^{\prime \prime}$ separation. Optical: No optical object lies coincident with, or between, the radio components. An extremely red star lies ' 20 " $\mathrm{S}$ of the radio source, and is associated with a stellar IRAS source (0.42 Jy at $12 \mu \mathrm{m}, 0.12 \mathrm{Jy}$ at $25 \mu \mathrm{m}$; Hacking \& Houck 1987). The star, listed in the HST Guide Star Catalog (GSC $4213596, V=9.4,180803.3,+660510$ ), has recently been classified as a K star by Kuemmel et al. (1998, private communication) using the $2.5 \mathrm{~m}$ Guillermo Haro telescope of INAOE, Mexico. 


\begin{tabular}{|c|c|c|c|c|c|c|c|c|c|c|c|c|c|c|c|}
\hline \multicolumn{5}{|c|}{ Radio Data } & \multicolumn{6}{|c|}{ X-ray Data } & \multicolumn{4}{|c|}{ Optical Data } & \multirow[t]{2}{*}{ Notes } \\
\hline VLA Source & $\alpha_{2000}$ & $\delta_{2000}$ & $S_{1.5}$ & & ROSAT source & $\Delta$ & $L_{\mathrm{oc}}$ & $M_{\mathrm{axL}}$ & $F_{\mathrm{x}}$ & & Class & $\Delta \alpha$ & $\Delta \delta$ & $B$ & \\
\hline $1808.8+6634$ & $18 \quad 849.7$ & 663430.4 & 26.2 & 0.8 & RXS J180850.2+663426 & 5 & $\mathrm{i}$ & 134 & 50.6 & 6.4 & $\mathrm{~S}$ & -1 & -1 & $17.5 \mathrm{~b}$ & 29 \\
\hline $1809.0+6704$ & $\begin{array}{lll}18 & 9 & 0.6\end{array}$ & $67 \quad 425.4$ & 17.7 & 0.6 & RXS J180859.7+670427 & 5 & $\mathrm{i}$ & 20 & 19.3 & 5.3 & $\mathrm{~S}$ & 1 & 0 & $20.5 \mathrm{~b}$ & 30 \\
\hline $1815.4+6806$ & 181524.0 & $\begin{array}{lll}68 & 626.6\end{array}$ & 381.4 & 12.2 & RXS J181521.5+680639 & 19 & $\mathrm{i}$ & 54 & 62.2 & 12.6 & $\mathrm{G}$ & 5 & 5 & 17.8 & 31 \\
\hline $1816.2+6627$ & 181611.6 & $\begin{array}{lll}66 & 27 & 3.7\end{array}$ & 3.1 & 0.1 & RXS J181611.2+662713 & 10 & $\mathrm{i}$ & 6 & 10.1 & 4.8 & $\mathrm{G}$ & 26 & 33 & $18.5 \mathrm{c}$ & 32 \\
\hline $1818.1+6349$ & $1818 \quad 7.8$ & 634951.6 & 20.9 & 1.0 & RXS J181805.8+634948 & 14 & o & 6 & 6.5 & 2.9 & $\mathrm{E}$ & $\ldots$ & $\ldots$ & $>22.5$ & 33 \\
\hline $1819.6+6857$ & 181937.6 & 685725.1 & $\cdots$ & $\cdots$ & RXS J181951.4+685704 & 77 & o & 24 & 93.2 & 11.2 & $\cdots$ & $\ldots$ & $\ldots$ & $\ldots$ & 34 \\
\hline $1819.7+6856$ & 181942.4 & 685652.6 & 735.3 & 22.1 & RXS J181951.4+685704 & 50 & o & 24 & 93.2 & 11.2 & $\mathrm{Gp}$ & $\ldots$ & $\ldots$ & 15.0 & 34 \\
\hline $1821.8+6818$ & 182149.0 & 681848.7 & $\cdots$ & $\cdots$ & RXS J182155.7+681852 & 38 & o & 15 & 14.9 & 4.3 & $\cdots$ & $\ldots$ & $\ldots$ & $\ldots$ & 35 \\
\hline $1822.0+6818$ & 182159.3 & $6818 \quad 43.4$ & 185.9 & 6.2 & RXS J182155.7+681852 & 22 & o & 15 & 14.9 & 4.3 & $\mathrm{~S}$ & 1 & -1 & $21.0 \mathrm{c}$ & 35 \\
\hline $1822.1+6637$ & $1822 \quad 3.5$ & 663714.8 & 10.7 & 0.5 & RXS J182203.6+663713 & 1 & $\mathrm{o}$ & 6 & 4.9 & 2.2 & $\mathrm{G}$ &. & $\ldots$ & 14.0 & 36 \\
\hline $1826.6+6706$ & 182637.4 & $\begin{array}{lll}67 & 6 & 44.4\end{array}$ & 107.6 & 3.6 & RXS J182636.9+670645 & 3 & o & 38 & 34.0 & 5.5 & $\mathrm{G}$ & 0 & 0 & 17.7 & 37 \\
\hline
\end{tabular}

${ }^{29} 1808.8+6634$ Radio: possibly extended, $36^{\prime \prime}$

${ }^{30} 1809.0+6704$ Radio: may be northern component of triple source with $1808.9+6703$ and 1809.0+6702, extent 54" . Contour map in Kollgaard et al. (1994).

${ }^{31} 1815.4+6806=8 \mathrm{C} 1815+680=4 \mathrm{C} 68.20$. Radio: extended, $48^{\prime \prime}$. Optical: $R=17.8 \mathrm{mag}$ broad line radio galaxy at $z=0.23$ (Veron). Numerous $B \quad 20$ stars or galaxies nearby; radio galaxy may be a member of a group, but X-ray source unlikely to be group emission.

${ }^{32} 1816.2+6627$ Radio: extended $36^{\prime \prime} \mathrm{SE}-\mathrm{NW}$. Contour map in Kollgaard et al. (1994). Optical: The NW portion coincides with the $V=10.5$ K0 star BD $+66^{\circ} 1092$ (SIMBAD). The SE portion coincides with a $B=18.5 \mathrm{c}$ galaxy. The table refers to the latter identification.

${ }^{33} 1818.1+6349$ Radio: This source appears to be the SE lobe of a multiple-component source $3-4^{\prime}$ in extent. Contour map in Kollgaard et al. (1994). Optical: Several $B=17-18$ stars or galaxies lie around the radio sources, one of which coincides with radio component $1817.8+6351$. Given these uncertainties, we classify this as an empty field.

${ }^{34} 1819.6+6857,1819.6+6856$ and $1819.7+6856=4 \mathrm{C} 68.21$. X-ray: extended $\left(\mathrm{ML}_{\mathrm{ext}}=28.9\right)$. Radio: triple source with $54^{\prime \prime}$ extent, head-tail with core morphology located in the outer region of the extended X-ray structure. Optical: 15.0 mag galaxy in Abell 2304 with $z=0.0881$ (NED) is coincident with easternmost radio component (radio core). Abell 2304 has Richness Class 0, Bautz-Morgan Class II, and Distance Class 5 (Abell et al. 1989). (Fig. 5j).

${ }^{35} 1821.8+6818$ and $1822.0+6818$ Radio: probable double, $60^{\prime \prime}$ separation. Optical: a $B=21.0$ stellar object coincides with the brighter component.

${ }^{36} 1822.1+6637=$ NGC $6636=$ VV $679=$ IRAS 18220+6635. Radio: extended 24", brightest at center and located between the optical galaxies. Optical: 14 mag SBc Seyfert 2 galaxy with $z=0.0133$ with 16 mag compact galaxy companion (NED).

${ }^{37} 1826.6+6706=7 \mathrm{C}$ 1826+6704. Radio: possibly extended, 48". Optical: 17.7 mag galaxy with $z=0.287$ (NED). 


\begin{tabular}{|c|c|c|c|c|c|c|c|c|c|c|c|c|c|c|c|}
\hline \multicolumn{5}{|c|}{ Radio Data } & \multicolumn{6}{|c|}{ X-ray Data } & \multicolumn{4}{|c|}{ Optical Data } & \multirow[t]{2}{*}{ Notes } \\
\hline VLA Source & $\alpha_{2000}$ & $\delta_{2000}$ & $S_{1.5}$ & & ROSAT source & $\Delta$ & $L_{\mathrm{oc}}$ & $M_{\mathrm{axL}}$ & $F_{\mathrm{x}}$ & & Class & $\Delta \alpha$ & $\Delta \delta$ & $B$ & \\
\hline $1739.2+6907$ & 173914.1 & $\begin{array}{lll}69 & 743.3\end{array}$ & 2.7 & 0.2 & RXS J173917.3+690828 & 48 & o & 5 & 8.0 & 3.3 & $\mathrm{E}$ & $\cdots$ & $\cdots$ & $>22.5$ & \\
\hline $1744.9+6642$ & 174453.5 & 664253.7 & 29.8 & 1.1 & RXS J174458.3+664339 & 54 & $\mathrm{i}$ & 6 & 7.9 & 3.8 & $\mathrm{E}$ & $\cdots$ & $\ldots$ & $>22.5$ & \\
\hline $1745.7+6620$ & 174544.0 & 662014.6 & 2.5 & 0.2 & RXS J174546.7+662056 & 44 & $\mathrm{i}$ & 6 & 7.5 & 3.4 & $\mathrm{E}$ & . & $\cdots$ & $>22.5$ & \\
\hline $1747.7+6345$ & 174742.4 & $6345 \quad 5.9$ & 1.2 & 0.2 & iRXS J174734.0+634511 & 56 & o & 10 & 20.0 & 5.1 & $\mathrm{G}$ & -3 & -2 & $21.5 \mathrm{~b}$ & \\
\hline $1749.2+6632$ & 174912.2 & $6632 \quad 8.6$ & 1.5 & 0.1 & RXS J174918.3+663146 & 42 & $\mathrm{i}$ & 16 & 11.2 & 3.4 & $\mathrm{E}$ & $\cdots$ & $\ldots$ & $>22.5$ & \\
\hline $1749.5+6726$ & 174927.0 & 672625.3 & 1.8 & 0.1 & RXS J174934.5+672554 & 53 & $\mathrm{i}$ & 6 & 8.1 & 3.6 & $\mathrm{E}$ & . & $\cdots$ & $>22.5$ & \\
\hline $1751.0+6535$ & $1751 \quad 0.9$ & 653554.2 & 1.1 & 0.1 & RXS J175106.6+653559 & 35 & $\mathrm{i}$ & 6 & 9.5 & 4.1 & $\mathrm{E}$ & .. & $\ldots$ & $>22.5$ & \\
\hline $1753.2+6619$ & 175313.7 & 661949.8 & 8.0 & 1.0 & RXS J175313.0+662020 & 31 & $\mathrm{i}$ & 10 & 6.7 & 2.3 & $\mathrm{E}$ & $\cdots$ & $\cdots$ & $>22.5$ & \\
\hline $1755.2+6505$ & $1755 \quad 9.6$ & 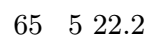 & 15.7 & 0.7 & RXS J175519.8+650451 & 71 & $\mathrm{i}$ & 34 & 30.4 & 6.6 & $\mathrm{~S}$ & 6 & -1 & $>22.5$ & 1 \\
\hline $1756.2+6807$ & 175611.7 & $\begin{array}{lll}68 & 7 & 59.1\end{array}$ & 2.8 & 0.1 & RXS J175614.4+680712 & 49 & $\mathrm{o}$ & 336 & 77.5 & 5.0 & $\mathrm{E}$ & 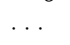 & $\cdots$ & $>22.5$ & \\
\hline $1756.7+6531$ & 175640.1 & 653146.2 & 56.5 & 1.8 & RXS J175641.9+653123 & 26 & $\mathrm{i}$ & 7 & 7.4 & 2.9 & $\mathrm{~S}$ & 2 & 0 & $22.0 \mathrm{c}$ & \\
\hline $1756.8+6546$ & 175645.6 & 654622.3 & 14.7 & 0.6 & RXS J175651.7+654601 & 43 & $\mathrm{i}$ & 7 & 5.9 & 2.4 & $\mathrm{E}$ & $\cdots$ & $\cdots$ & $>22.5$ & \\
\hline $1757.1+6353$ & $1757 \quad 7.3$ & 635315.4 & 1.0 & 0.1 & RXS J175710.1+635235 & 44 & o & 43 & 21.6 & 3.6 & $\mathrm{E}$ & $\cdots$ & $\ldots$ & $>22.5$ & \\
\hline $1757.4+6536$ & 175723.3 & 653610.4 & 45.6 & 1.8 & RXS J175728.8+653539 & 46 & $\mathrm{i}$ & 8 & 6.7 & 2.6 & $\mathrm{~S}$ & 4 & 0 & $22.0 \mathrm{a}$ & \\
\hline $1758.1+6408$ & $1758 \quad 6.6$ & $64 \quad 854.6$ & 1.5 & 0.1 & RXS J175801.2+640932 & 52 & o & 538 & 127.0 & 6.4 & $\mathrm{E}$ & 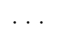 & $\ldots$ & $>22.5$ & 2 \\
\hline $1758.2+6729$ & 175812.1 & 672927.3 & 13.7 & 0.5 & RXS J175813.8+673006 & 41 & $\mathrm{i}$ & 6 & 6.1 & 2.5 & $\mathrm{E}$ & $\cdots$ & $\ldots$ & $>22.5$ & \\
\hline $1758.8+6607$ & 175850.5 & $\begin{array}{lll}66 & 7 & 15.6\end{array}$ & 1.4 & 0.1 & RXS J175853.3+660637 & 41 & $\mathrm{i}$ & 9 & 4.6 & 1.6 & G & -3 & -1 & $18.0 \mathrm{c}$ & 3 \\
\hline $1759.4+6735$ & 175923.0 & 673555.1 & 11.9 & 0.4 & RXS J175919.8+673628 & 38 & $\mathrm{i}$ & 7 & 9.4 & 3.3 & $\mathrm{E}$ & $\ldots$ & $\ldots$ & $>22.5$ & \\
\hline $1800.3+6739$ & $\begin{array}{lll}18 & 0 & 16.1\end{array}$ & 673921.9 & 8.9 & 0.3 & RXS J180013.4+673835 & 49 & $\mathrm{i}$ & 11 & 8.6 & 3.0 & $\mathrm{E}$ & $\cdots$ & $\cdots$ & $>22.5$ & \\
\hline $1801.5+6645$ & $18 \quad 132.5$ & $\begin{array}{lll}66 & 45 & 8.6\end{array}$ & 3.6 & 0.3 & RXS J180137.7+664526 & 36 & $\mathrm{i}$ & 10 & 3.4 & 1.0 & $\mathrm{Gp}$ & $\cdots$ & $\cdots$ & $>22.5$ & 4 \\
\hline $1802.9+6450$ & $18 \quad 256.0$ & 645059.7 & 1.1 & 0.1 & RXS J180301.6+645028 & 47 & $\mathrm{i}$ & 8 & 7.6 & 3.1 & $\mathrm{E}$ & $\ldots$ & $\ldots$ & $>22.5$ & \\
\hline $1805.2+6737$ & $\begin{array}{lll}18 & 5 & 9.5\end{array}$ & 673746.5 & 3.3 & 0.1 & RXS J180514.9+673738 & 32 & $\mathrm{i}$ & 8 & 10.8 & 401 & $\mathrm{E}$ & $\cdots$ & $\cdots$ & $>22.5$ & \\
\hline $1806.4+6619$ & $18 \quad 625.1$ & 661957.4 & 1.3 & 0.2 & RXS J180616.7+662009 & 52 & $\mathrm{i}$ & 5 & 4.7 & 2.0 & $\mathrm{E}$ & $\cdots$ & $\cdots$ & $>22.5$ & 5 \\
\hline $1807.5+6557$ & $18 \quad 731.1$ & 655719.3 & 2.7 & 0.1 & RXS J180723.7+655640 & 60 & $\mathrm{i}$ & 14 & 10.7 & 3.3 & $\mathrm{E}$ & ... & $\ldots$ & $>22.5$ & \\
\hline $1807.9+6617$ & $18 \quad 753.0$ & 661730.3 & 21.6 & 1.0 & RXS J180747.6+661737 & 34 & $\mathrm{i}$ & 85 & 30.6 & 4.6 & $\mathrm{E}$ & $\cdots$ & $\cdots$ & $>22.5$ & \\
\hline $1808.8+6607$ & $18 \quad 849.8$ & $\begin{array}{lll}66 & 7 & 8.5\end{array}$ & 4.0 & 0.2 & RXS J180844.8+660624 & 53 & $\mathrm{i}$ & 7 & 6.5 & 2.6 & $\mathrm{E}$ & $\cdots$ & $\cdots$ & $>22.5$ & \\
\hline $1810.0+6345$ & $18 \quad 957.2$ & $6345 \quad 2.6$ & 44.5 & 2.1 & RXS J181003.7+634426 & 56 & o & 56 & 36.3 & 5.1 & $\mathrm{E}$ & $\cdots$ & $\cdots$ & $>22.5$ & \\
\hline $1810.1+6634$ & $1810 \quad 6.1$ & 663414.4 & 2.2 & 0.1 & RXS J181006.1+663321 & 53 & $\mathrm{i}$ & 8 & 8.5 & 3.3 & $\mathrm{E}$ & $\cdots$ & $\cdots$ & $>22.5$ & \\
\hline $1811.0+6810$ & 181059.2 & 681029.0 & 9.2 & 0.7 & RXS J181106.8+681044 & 45 & o & 5 & 5.2 & 2.3 & $\mathrm{E}$ & $\cdots$ & $\cdots$ & $>22.5$ & \\
\hline $1812.9+6533$ & 181252.6 & 653344.7 & 1.4 & 0.1 & RXS J181246.2+653350 & 40 & $\mathrm{i}$ & 30 & 24.5 & 6.1 & $\mathrm{E}$ & $\cdots$ & $\cdots$ & $>22.5$ & \\
\hline $1813.5+6641$ & 181330.0 & 664155.9 & 1.8 & 0.2 & RXS J181339.3+664132 & 60 & $\mathrm{i}$ & 5 & 8.2 & 3.8 & $\mathrm{E}$ & $\cdots$ & $\cdots$ & $>22.5$ & 6 \\
\hline $1819.9+6749$ & 181955.5 & 674910.3 & 15.9 & 0.6 & RXS J181952.8+674820 & 52 & o & 6 & 8.4 & 3.4 & $\mathrm{G}$ & 5 & -1 & $19.5 \mathrm{c}$ & \\
\hline
\end{tabular}

${ }^{1} 1755.2+6505$ Radio: possibly extended, $36^{\prime \prime}$

${ }^{2} 1758.1+6408$ Radio/X-ray offset exceeds 40" . X-ray: very bright. Radio: faint and unresolved. Optical: X-ray position coincides with unstudied $V=10.6$ G star $\mathrm{BD} 64^{\circ} 1235=\mathrm{SAO} 17709$ (SIMBAD)

${ }^{3} 1758.8+6607$ Radio/X-ray offset exceeds $40^{\prime \prime}$. Radio: faint and unresolved. Optical: IRAS detected starburst galaxy $(0.07 \mathrm{Jy}$ at $60 \mu \mathrm{m}$ and $0.11 \mathrm{Jy}$ at $100 \mu \mathrm{m})$ with $z=0.0799$, member of massive supercluster (Ashby et al. 1996).

${ }^{4}$ 1801.5+6645 Optical: associated with NEPX1, an X-ray-selected galaxy cluster with $z=0.09$ (Burg et al. 1992; Hasinger et al. 1991) (Fig. 5h).

${ }^{5} 1806.4+6619$ Possibly associated with $I R A S$-detected triple galaxy, $0.6^{\prime}$ distant $(z=0.107 ; 0.09 \mathrm{Jy}$ at $60 \mu \mathrm{m}$ and $<0.1 \mathrm{Jy}$ at $100 \mu \mathrm{m} ;$ Ashby et al. 1996$)$.

${ }^{6} 1813.5+6641=$ IRAS F18135+6641. 